\title{
Auction and the Informed Seller Problem*
}

\author{
B. Jullien ${ }^{\dagger}$ \\ T. Mariotti ${ }^{\ddagger \S}$
}

August 2002

revised October 2004

\begin{abstract}
A seller possessing private information about the quality of a good attempts to sell it through a second-price auction with announced reserve price. The choice of a reserve price transmits information to the buyers. We compare the outcome of a signalling game in which the seller runs the auction himself and a screening game in which a monopoly broker chooses the trading mechanism. In the former case, we characterize the equilibria of the resulting signalling game and show that they lead to reduced levels of sale compared to the symmetric information situation. We compare the unique separating equilibrium with the outcome that would be chosen by the monopoly broker. The ex-ante expected probability of trade may be larger with a monopoly broker, as well as the ex-ante total expected surplus.
\end{abstract}

${ }^{*}$ We wish to thank Bruno Biais, Bernard Caillaud, David Martimort, Benny Moldovanu and Jean-Charles Rochet, as well as seminar participants at the universities of Bristol, Paris I, Salerno and Toulouse for insightful discussions. We are also grateful to two anonymous referees and an associate editor for helpful comments and suggestions.

${ }^{\dagger}$ GREMAQ-IDEI, Université de Toulouse I, 21 Allée de Brienne, 31000 Toulouse, France.

${ }^{\ddagger}$ GREMAQ-IDEI, Université de Toulouse I, 21 Allée de Brienne, 31000 Toulouse, France.

${ }^{\S}$ Corresponding author. E-mail addresses: bjullien@cict.fr (B. Jullien), mariotti@cict.fr (T. Mariotti). 


\section{Introduction}

In this paper, we consider an auction market subject to a lemon problem (Akerlof, 1970). We derive the equilibrium outcome of a decentralized market structure, and compare it with the outcome of a trading process centralized by a monopoly broker. The lemon problem arises because the seller, who designs the auction in the decentralized structure, has access to some non-verifiable information relevant to buyers. This results in an informed principal situation (Maskin and Tirole, 1992) which implies sub-optimal trade: sellers signal the quality of the object put to sale by raising the reserve price. In the centralized market structure, the monopoly broker acts as a pure intermediary, with no particular expertise. But because all trades have to be conducted through his house, he is able to design screening mechanisms that induce revelation of the seller's information to the buyers, thereby limiting the lemon problem. When the efficiency loss due to monopoly distortion is not too large, the monopoly broker generates more trade and larger total surplus than the decentralized structure.

Traditional auction theory considers auctions conducted by sellers and attempts to derive the revenue maximizing or the efficient auction. In practice, however, instances where the seller chooses and conducts himself the auction - such as public procurement or treasury bond auctions - are not so numerous. By large, many auctions or auction-like mechanisms in the private sector are conducted by intermediaries - such as for instance in IPOs, or for the sale of art items, wine or real estate. The spectacular success of eBay has pointed to the potential of developing auction-like trading mechanisms when the cost of gathering and processing information is reduced, as it is the case for Internet. One noticeable feature of eBay is that it has being successful in solving quality issues for secondary goods through adequate mechanism design and information feedback (Dellarocas, 2004).

In their seminal work on efficient bilateral mechanisms, Myerson and Satterthwaite (1983) studied from an auction perspective the problem faced by a broker who intermediates trades. Since then, little attention has been paid in the auction literature on the role of intermediaries. Aside auction theory, however, many authors have emphasized that intermediation may be instrumental in reducing information related costs. The literature has mostly focused on the expertise of intermediaries. Indeed, it is typically assumed that intermediaries have access to some information technology that raises the efficiency of market organization. For instance, they may facilitate the search of a trading partner (Rubinstein and Wolinsky, 1987, Yavas, 1994, Caillaud and Jullien, 2003). Alternatively, they may have expertise in certification, screening or monitoring (Diamond 1984, Biglaiser, 1993). In our setup, the monopoly broker has no expertise except in conducting auctions, and this is precisely the source of the efficiency gains. ${ }^{1}$ Indeed, an informed intermediary may face similar issues as an informed seller since he may not have the proper incentives to reveal the information to the market at the interim stage (Lizzeri, 1999, Peyrache and Quesada, 2003). By contrast, being uninformed, our monopoly broker is not constrained by the fact that some choice of trading mechanism may convey a bad signal about the value of the traded object, which is the source of the results.

The starting point of our analysis is the decentralized structure in which a seller who owns an indivisible object directly faces two potential buyers. The seller's valuation for the good is private information. Each buyer's ex-post valuation for the good consists of a common value component that is proportional to the seller's valuation, and of a private value component which is private information. One interpretation is that the seller knows the quality of the object for sale, and that the buyers' valuations depend on the quality of the object as well

\footnotetext{
${ }^{1}$ Assuming a monopoly intermediary means however that some type of exclusive expertise or specific asset prevents entry in the intermediation market.
} 
as on idiosyncratic preference shocks. In a decentralized market, the seller chooses a reserve price and organizes a second-price sealed bid auction with public reserve price. This informed seller game thus amounts to a signalling game, where the reserve price chosen by the seller plays the role of a signal of the quality of the object, and the buyers react to this by adjusting their bids in the auction. ${ }^{2}$

Surprisingly, this issue of the informed seller in the context of an auction has not yet been resolved. Milgrom and Weber (1982) show that a seller always benefits from revealing information that is affiliated to the buyers' valuations, but this linkage principle holds ex-ante for verifiable information, while we analyze the problem at the interim stage for non-verifiable information that is correlated with the seller's opportunity cost of selling. The corresponding signalling game does not satisfy standard assumptions. In particular, for a given value of the reserve price, the expected utility of the seller need not be an increasing function of the buyers' beliefs about the quality of the object.

Adapting Mailath's (1987) techniques, we characterize the unique separating equilibrium of the informed seller game. ${ }^{3}$ Next, we study equilibria with monotone beliefs, in which the buyers' beliefs are increasing with respect to the reserve price chosen by the seller on and off the equilibrium path. While this assumption of monotone beliefs would not be restrictive in a standard signalling game, this is not so in the informed seller game. The main result is that, in line with Akerlof (1970), equilibria with monotone beliefs are characterized by underselling relative to the buyer's beliefs: for any realization of the seller's information about the quality of the object for sale, the equilibrium probability of sale is lower than the probability of sale that would be optimal given the quality of the object and the corresponding equilibrium buyers' beliefs. An important consequence of this result is that the probability of sale is uniformly lower than in the public information benchmark in which the quality of the object for sale is common knowledge. The assumption of monotone beliefs is crucial for this result. Indeed, we provide examples of equilibria with non monotone beliefs such that, for some realization of the seller's information, the equilibrium probability of trade is larger than in the public information benchmark.

We then turn to the monopoly broker problem. This is a standard screening problem, and the optimal mechanism can be interpreted as one in which the broker organizes a second-price sealed bid auction on behalf of the seller, letting the seller announce a reserve price in exchange for a contingent fee. The announced reserve price then reveals the seller's information to the buyers. We compare the separating decentralized allocation with the monopoly broker allocation. At the interim stage, it is not possible to rank the two allocations, as the broker sells more often for low seller's valuations, but less often for high seller's valuations. ${ }^{4}$ However, from an ex-ante perspective, we prove that provided the uncertainty on the seller's information is not too large, the expected probability of sale is larger when trade is intermediated by a monopoly broker. An analogous result holds true for the ex-ante expected surplus.

The paper is organized as follows. The informed seller game is described in Section 2.

\footnotetext{
${ }^{2}$ Notice that in the last stage of the informed seller game, that is in the auction itself, buyers share the same information on the common value component. Hence the auction is basically an independent private value one and revenue equivalence holds. Provided that the seller is restricted to choosing a selling mechanism and that he is not an active participant of the mechanism once this choice is made (as may occur in a double auction), there is no loss of generality in assuming a second-price sealed bid auction.

${ }^{3}$ In contemporaneous work, Cai, Riley and Ye (2002) solve for the separating equilibrium of a signaling game in which a seller chooses a mark-up over the buyers' beliefs. The reserve price is then defined as the sum of the beliefs and the mark-up. This appears to be close to our resolution of the separating equilibrium of the game where the seller chooses directly the reserve price.

${ }^{4}$ This is true more generally for any equilibrium with monotone beliefs.
} 
In Section 3, we provide some basic properties of equilibria. Section 4 presents the unique separating equilibrium, while equilibria with monotone beliefs are characterized in Section 5. Section 6 is devoted to the monopoly broker allocation, and our comparison result is stated in Section 7. Section 8 concludes. All proofs are in the Appendix.

\section{The informed seller game}

\subsection{The model}

There are three risk-neutral agents, one seller and two buyers, and one object for sale initially owned by the seller. We denote by $\tilde{\theta}$ the valuation of the seller for this object, and by $\tilde{\theta}^{1}$ and $\tilde{\theta}^{2}$ those of the buyers. It is assumed that for some $\lambda \in(0,1)$, one has, for each $i=1,2$ :

$$
\tilde{\theta}^{i}=\lambda \tilde{\theta}+(1-\lambda) \tilde{\zeta}^{i},
$$

where $\tilde{\theta}$ and $\tilde{\zeta}^{i}, i=1,2$, are independently distributed, with continuously differentiable distribution functions $G$ and $F$ with support $[0,1]$. The corresponding density functions $g$ and $f$ are taken to be strictly positive, and to satisfy the usual monotone hazard rate properties, namely that $G / g$ is strictly increasing and $(1-F) / f$ is strictly decreasing. One interpretation of (1) is that the buyers' valuations depend on the underlying quality of the object, $\tilde{\theta}$, as well as on idiosyncratic preference shocks, $\tilde{\zeta}^{i}, i=1,2$.

It is assumed that $\tilde{\theta}$ and $\tilde{\zeta}^{i}, i=1,2$, are private information of the seller and of buyer $i=1,2$, respectively. Following standard usage, these parameters are referred to as the seller's and the buyers' types. Since $\tilde{\theta}$ and $\tilde{\zeta}^{i}, i=1,2$, are independently distributed, buyers have the same beliefs about the seller's type.

The informed seller game can then be described as follows. First, the seller puts the object for sale by running a second-price sealed bid auction with an announced reserve price. Next, upon observing the reserve price chosen by the seller, buyers form beliefs about the seller's type, and then bid accordingly.

\subsection{The expected utility of the seller}

For any reserve price $r$ and induced beliefs $\varphi$ about the expectation of the seller's type, define:

$$
z(\varphi, r)=\frac{r-\lambda \varphi}{1-\lambda}
$$

Then, according to (1), trade occurs if at least one buyer $i=1,2$ has a type $\zeta^{i} \geq z(\varphi, r)$. The probability of no trade is thus:

$$
\pi(\varphi, r)=F(z(\varphi, r))^{2} .
$$

Given a reserve price $r$ and induced beliefs $\varphi$, the expected utility of a seller with type $\theta$ in a second-price sealed bid auction can then be easily computed as:

$$
U(\theta, \varphi, r)=\theta+\lambda(\varphi-\theta)[1-\pi(\varphi, r)]+(1-\lambda) \int_{z(\varphi, r)}^{1}\left[\zeta-\frac{1-F(\zeta)}{f(\zeta)}-\theta\right] \mathrm{d} F(\zeta)^{2} .
$$

Note that (4) reduces to the usual formula in the standard private values situation where $\lambda=0$ (Riley and Samuelson, 1981). According to (4), the expected gains from trade $U(\theta, \varphi, r)-\theta$ consist of two components. The first,

$$
(1-\lambda) \int_{z(\varphi, r)}^{1}\left[\zeta-\frac{1-F(\zeta)}{f(\zeta)}-\theta\right] \mathrm{d} F(\zeta)^{2}
$$


represents the expected gains that the seller would obtain if his type were common knowledge, so that $\varphi=\theta$, in a second-price sealed bid auction in which the reserve price is adjusted to $\lambda \theta+(1-\lambda) z(\varphi, r)$ so as to maintain the probability of no trade $\pi(\varphi, r)$. The second,

$$
\lambda(\varphi-\theta)[1-\pi(\varphi, r)]
$$

represents the expected gains from manipulating the buyers' beliefs. It reflects that for a given probability of no trade, an upward bias in beliefs would correspond to a higher reserve price, along with higher bids. Specifically, the bids would be raised by an amount $\lambda(\varphi-\theta)$, as well as the reserve price that maintains unchanged the probability of no trade.

As illustrated by this discussion, one of the difficulties raised by the informed seller game is that, while we may derive intuitions about how the probability of no trade affects the expected payoff of the seller, there is no straightforward relationship between the reserve price and the probability of no trade, as the link between the two ultimately depends on buyers' beliefs, which are determined in equilibrium.

\subsection{A useful change of variable}

Despite these reservations, it will be necessary for the analysis to transform the original formulation of the game, because the representation (4) for the expected utility of the seller is not convenient for analytical purposes. Indeed, the informed seller game as such does not satisfy standard assumptions. Specifically, the utility of a seller with type $\theta$ need not be increasing with respect to beliefs $\varphi$ for any given reserve price $r{ }^{5}$

The change of variable we propose relies on the fact that $U(\theta, \varphi, r)$ depends on the reserve price $r$ only through the probability of no trade, $\pi(\varphi, r)$. Indeed, by $(2)-(3), U(\theta, \varphi, r)=$ $U(\theta, \varphi, \lambda \varphi+(1-\lambda) z(\varphi, r))$ and $z(\varphi, r)=\left(F^{2}\right)^{-1}(\pi(\varphi, r))$. It is therefore natural to introduce an auxiliary function,

$$
\hat{U}(\theta, \varphi, \pi)=U\left(\theta, \varphi, \lambda \varphi+(1-\lambda)\left(F^{2}\right)^{-1}(\pi)\right),
$$

that measures the expected utility of the seller as a function of his type, the buyers' beliefs and the probability of no trade. In what follows we shall use the notation $z$ or $\pi$ whenever convenient, with the convention that $\pi=F(z)^{2}$. From (4) and (7), one can derive the following explicit expression for $\hat{U}$ :

$$
\hat{U}(\theta, \varphi, \pi)=\theta \pi+\lambda \varphi(1-\pi)+(1-\lambda) \int_{z}^{1}\left[\zeta-\frac{1-F(\zeta)}{f(\zeta)}\right] \mathrm{d} F(\zeta)^{2}
$$

It is clear from $(8)$ that, for $\pi \neq 1, \hat{U}(\theta, \varphi, \pi)$ is strictly increasing with respect to $\varphi$. Moreover, since $z$ is strictly increasing with respect to $\pi$, it follows from the monotone hazard rate property for $F$ that $\hat{U}(\theta, \varphi, \pi)$ is strictly concave with respect to $\pi$. Finally, the single-crossing condition is satisfied since, for $\pi \neq 1$,

$$
\frac{\hat{U}_{3}(\theta, \varphi, \pi)}{\hat{U}_{2}(\theta, \varphi, \pi)}=\frac{\theta-\lambda \varphi-(1-\lambda)\left[z-\frac{1-F(z)}{f(z)}\right]}{\lambda(1-\pi)}
$$

\footnotetext{
${ }^{5}$ Indeed, it is easy to verify that:

$$
U_{2}(\theta, \varphi, r)=\frac{\lambda}{1-\lambda}\left\{2 f(z(\varphi, r)) F(z(\varphi, r))(r-\theta)+(1-\lambda)[1-F(z(\varphi, r))]^{2}\right\},
$$

which may be negative if $r<\theta$. In Example 2 of Section 4, we construct an equilibrium of the informed seller game such that, with positive probability, the seller sets a reserve price below his own valuation for the object.
} 
is strictly increasing with respect to $\theta$. These convenient properties will be used repeatedly in the analysis. In particular, it will be helpful to study an auxiliary game in which the seller directly chooses the probability of no trade $\pi$, and has expected utility $\hat{U}(\theta, \varphi, \pi)$. This auxiliary game satisfies standard assumptions, unlike the original game. However, caution is required when translating results that are valid for the auxiliary game into results that are valid for the original game. Indeed, one cannot simply proceed as if the seller were directly choosing the probability of no trade instead of the reserve price, as this would effectively amount to select the buyers' beliefs.

\subsection{The symmetric information benchmark}

As a benchmark, it is helpful to consider the case in which the seller's type is common knowledge. For this purpose, let us introduce the mapping:

$$
\hat{\pi}(\theta, \varphi)=\underset{\pi \in[0,1]}{\arg \max } \hat{U}(\theta, \varphi, \pi),
$$

that gives the optimal probability of no trade for a seller of type $\theta$, given fixed buyers' beliefs $\varphi$. The mapping $\hat{\pi}$ is well-defined since $\hat{U}(\theta, \varphi, \pi)$ is strictly concave with respect to $\pi$ for any $(\theta, \varphi) \in[0,1]^{2}$. Let $\hat{z}(\theta, \varphi)=\left(F^{2}\right)^{-1}(\hat{\pi}(\theta, \varphi))$ be the corresponding minimum buyers' type for which trade can occur. It follows then from (2) that if the seller's type $\theta$ were common knowledge, so that $\varphi=\theta$, the optimal reserve price would be $\lambda \theta+(1-\lambda) \hat{z}(\theta, \theta)$, where $\hat{z}(\theta, \theta)$ is the unique solution to the equation:

$$
\zeta-\frac{1-F(\zeta)}{f(\zeta)}=\theta
$$

which implicitly defines the optimal reserve price in a second-price sealed bid auction with private values in which the valuations of the buyers are given by $\tilde{\zeta}^{i}, i=1,2$ (Riley and Samuelson, 1981). It will be assumed that $\hat{z}(\theta, \theta)$ is a differentiable function of $\theta$, with a uniformly bounded derivative over $[0,1]$.

\section{Preliminaries}

We start with some basic properties of equilibria. In the informed seller game, a perfect Bayesian equilibrium, or equilibrium for short, is a pair of functions $\left(\phi^{*}, r^{*}\right):[0,1]^{2} \rightarrow[0,1]^{2}$ that satisfy the sequential rationality condition:

$$
U\left(\theta, \phi^{*}\left(r^{*}(\theta)\right), r^{*}(\theta)\right) \geq U\left(\theta, \phi^{*}(r), r\right)
$$

for every $(\theta, r) \in[0,1]^{2}$, along with the Bayesian rationality condition:

$$
\phi^{*}(r)=\mathrm{E}\left[\tilde{\theta} \mid r^{*}(\tilde{\theta})=r\right]
$$

for every $r$ in the range of $r^{*}$. Whenever the type of the seller is $\theta$, the buyers' beliefs in equilibrium are given by $\varphi^{*}(\theta)=\phi^{*}\left(r^{*}(\theta)\right)$.

It is helpful to rewrite the equilibrium conditions in terms of the auxiliary function $\hat{U}$. In analogy with (2)-(3), define $z^{*}(\theta)=z\left(r^{*}(\theta), \varphi^{*}(\theta)\right)$ and $\pi^{*}(\theta)=F\left(z^{*}(\theta)\right)^{2}$. Bayesian rationality implies that if $r^{*}(\theta)=r^{*}\left(\theta^{\prime}\right)$, then $\varphi^{*}(\theta)=\varphi^{*}\left(\theta^{\prime}\right)$ and therefore $\pi^{*}(\theta)=\pi^{*}\left(\theta^{\prime}\right)$. For each $\theta \in[0,1]$, define $\hat{U}^{*}(\theta)=\hat{U}\left(\theta, \varphi^{*}(\theta), \pi^{*}(\theta)\right)$. Sequential rationality then implies that the incentive compatibility constraint:

$$
\hat{U}^{*}(\theta)=\max _{\theta^{\prime} \in[0,1]} \hat{U}\left(\theta, \varphi^{*}\left(\theta^{\prime}\right), \pi^{*}\left(\theta^{\prime}\right)\right)
$$


holds for every $\theta \in[0,1]$. The following result is a direct consequence of (11).

Lemma 1. In any equilibrium, $\pi^{*}$ and $\hat{U}^{*}$ are non-decreasing over $[0,1]$.

It follows in particular that in any equilibrium, the probability of trade is a decreasing function of the seller's type. Thus, in a manner reminiscent of Leland and Pyle (1977) or DeMarzo and Duffie (1999), the only way for the seller to credibly signal a high type is to accept a lower probability of trade, reflecting the underlying adverse selection problem.

A by-product of the proof of Lemma 1 is that $\hat{U}^{*}$ is almost everywhere differentiable over $[0,1]$. By the Envelope Theorem, it follows from (8) and (11) that:

$$
\frac{\mathrm{d} \hat{U}^{*}}{\mathrm{~d} \theta}(\theta)=\pi^{*}(\theta)
$$

for almost every $\theta \in[0,1]$.

A direct consequence of the envelope condition (12) is that setting a reserve price equal to the symmetric information reserve price $\lambda \theta+(1-\lambda) \hat{z}(\theta, \theta)$ for each $\theta \in[0,1]$ cannot constitute an equilibrium of the informed seller game. Indeed, with such an allocation, the seller's utility would be:

$$
\hat{U}(\theta, \theta, \hat{\pi}(\theta, \theta))=\theta+(1-\lambda) \int_{\hat{z}(\theta, \theta)}^{1}\left[\zeta-\frac{1-F(\zeta)}{f(\zeta)}-\theta\right] \mathrm{d} F(\zeta)^{2},
$$

and, from (10) and (13), a direct computation shows that:

$$
\frac{\mathrm{d} \hat{U}}{\mathrm{~d} \theta}(\theta, \theta, \hat{\pi}(\theta, \theta))=\lambda+(1-\lambda) \hat{\pi}(\theta, \theta) .
$$

For every $\theta \in[0,1)$, this coincides with $\hat{\pi}(\theta, \theta)$ only in the standard private values situation where $\lambda=0$. Whenever $\lambda>0$, this allocation violates the envelope condition (12), and is thus incompatible with an equilibrium of the informed seller game.

Another important consequence of Lemma 1 is that equilibrium reserve prices and beliefs are monotone functions of the seller's type.

Lemma 2. In any equilibrium, $r^{*}$ and $\varphi^{*}$ are non-decreasing over $\left\{\theta \in[0,1] \mid \pi^{*}(\theta)<1\right\}$.

The conclusion of Lemma 2 can be strengthened as follows. Whenever $\pi^{*}(\theta)=1$ in equilibrium, a seller of type $\theta$ may as well set a reserve price equal to 1 . Since the seller always have the option not to sell, this leaves the incentives of the other types of the seller unaffected. For convenience, one can therefore focus without loss of generality on equilibria such that $r^{*}$ and $\varphi^{*}$ are non-decreasing over the whole interval $[0,1]$. It follows that the equilibrium belief function $\phi^{*}$ is non-decreasing over the range of $r^{*}$. However, Lemma 2 says nothing about out-of-equilibrium beliefs, that is, on the global shape of $\phi^{*}$ outside the range of $r^{*}$. As we will see in Section 5, the characterization of equilibria is vastly simplified if we impose the further intuitive restriction that $\phi^{*}$ be non-decreasing over its entire domain.

\section{The separating allocation}

In this section, we characterize the unique separating allocation consistent with an equilibrium of the informed seller game. By (11), an incentive compatible separating allocation is given by a one-to-one mapping $\pi^{*}$ that satisfies:

$$
\theta \in \underset{\theta^{\prime} \in[0,1]}{\arg \max } \hat{U}\left(\theta, \theta^{\prime}, \pi^{*}\left(\theta^{\prime}\right)\right)
$$


for every $\theta \in[0,1]$. Note that (15) is the incentive compatibility condition for a separating allocation of the auxiliary game in which the seller directly chooses the probability of no trade $\pi$ and has expected utility $\hat{U}(\theta, \varphi, \pi)$. In order to be consistent with an equilibrium of the informed seller game, such an allocation must satisfy an appropriate boundary condition, as stated in the following lemma.

Lemma 3. In any separating equilibrium of the informed seller game,

$$
\pi^{*}(0)=\hat{\pi}(0,0)
$$

The boundary condition (16) expresses the fact that, in any separating equilibrium, there is no distortion at the bottom of the seller's type distribution relative to the symmetric information benchmark. Building on Mailath (1987), we shall prove that there exists a unique solution $\pi^{S}$ to (15)-(16). This solution is characterized by an intuitive differential equation, which reflects the first-order condition for (15):

$$
\lambda\left[1-\pi^{*}(\theta)\right]=(1-\lambda)\left[z^{*}(\theta)-\frac{1-F\left(z^{*}(\theta)\right)}{f\left(z^{*}(\theta)\right)}-\theta\right] \frac{\mathrm{d} \pi^{*}}{\mathrm{~d} \theta}(\theta),
$$

where $z^{*}(\theta)=\left(F^{2}\right)^{-1}\left(\pi^{*}(\theta)\right)$ for every $\theta \in[0,1]$. The left-hand side of (17) represents the expected marginal benefit for a seller of type $\theta$ of manipulating the buyers' beliefs by an infinitesimal amount $\mathrm{d} \theta$ by choosing a probability of no sale $\pi^{*}(\theta+\mathrm{d} \theta)$. The right-hand side of (17) represents the expected marginal cost of this manipulation in terms of increased probability of no trade. In equilibrium, the bracketed term on the right-hand side of (17) will be positive, as the probability of no trade $\pi^{*}(\theta)$ will be larger than the symmetric information probability of no trade $\hat{\pi}(\theta, \theta)$. One has the following result.

Proposition 1. There exists a unique separating allocation that is incentive compatible and satisfies the boundary condition (16). It is given by the unique strictly increasing solution $\pi^{S}$ to the following initial boundary value problem:

$$
\begin{aligned}
\frac{\mathrm{d} \pi^{*}}{\mathrm{~d} \theta}(\theta) & =\frac{\lambda\left[1-\pi^{*}(\theta)\right]}{(1-\lambda)\left[z^{*}(\theta)-\frac{1-F\left(z^{*}(\theta)\right)}{f\left(z^{*}(\theta)\right)}-\theta\right]}, \\
\pi^{*}(0) & =\hat{\pi}(0,0),
\end{aligned}
$$

where $z^{*}(\theta)=\left(F^{2}\right)^{-1}\left(\pi^{*}(\theta)\right)$ for every $\theta \in[0,1]$. In this allocation, the probability of no trade is strictly larger than the probability of no trade that would arise if the seller's type were common knowledge,

$$
\pi^{S}(\theta)>\hat{\pi}(\theta, \theta)
$$

for every $\theta \in(0,1)$.

The proof closely follows Mailath (1987). The only complication relative to his setup is that $\hat{U}_{2}$ can vanish at $\pi=1$. Special care must thus be taken in applying his results.

To turn the separating allocation $\pi^{S}$ into an equilibrium of the informed seller game, one needs only to compute the equilibrium reserve price and specify appropriate out-ofequilibrium beliefs. For any $\theta \in[0,1]$, let:

$$
r^{S}(\theta)=\lambda \theta+(1-\lambda) z^{S}(\theta)
$$


and for any $r \in[0,1]$, let:

$$
\phi^{S}(r)=\left\{\begin{array}{lll}
0 & \text { if } & r<r^{S}(0), \\
\theta & \text { if } & r=r^{S}(\theta) .
\end{array}\right.
$$

Given these beliefs, and no matter his type, the seller does not want to deviate to some reserve price $r<r^{S}(0)$. Indeed, for any $\theta \in[0,1], U(\theta, 0, r)$ is strictly increasing with respect to $r$ over $[0,(1-\lambda) \hat{z}(\theta, \theta)]$. Since $r^{S}(0)=(1-\lambda) \hat{z}(0,0)$ and $\hat{z}(\theta, \theta)$ is strictly increasing with respect to $\theta$, it follows that:

$$
\hat{U}\left(\theta, \theta, \pi^{S}(\theta)\right) \geq \hat{U}\left(\theta, 0, \pi^{S}(0)\right)=U\left(\theta, 0, r^{S}(0)\right)>U(\theta, 0, r)
$$

for any $r<r^{S}(0)$. Hence $\left(\phi^{S}, r^{S}\right)$ is a separating equilibrium of the informed seller game.

A natural question is whether $\pi^{S}$ is the only allocation compatible with a separating equilibrium of the informed seller game. Indeed, because the seller does not choose directly the probability of selling, one cannot a priori rule out equilibria in which $r^{*}$ would be one-toone, but $\pi^{*}$ would not. However, this can never arise over the interval of types $\theta$ such that $\pi^{*}(\theta)<1$. Indeed, suppose that $\pi^{*}(\theta)=\pi^{*}\left(\theta^{\prime}\right)<1$ for $\theta<\theta^{\prime}$ in a separating equilibrium. Then $\hat{U}\left(\theta, \varphi, \pi^{*}(\theta)\right)$ is strictly increasing with respect to $\varphi$. It would follow that:

$$
\hat{U}\left(\theta, \varphi^{*}\left(\theta^{\prime}\right), \pi^{*}\left(\theta^{\prime}\right)\right)=\hat{U}\left(\theta, \theta^{\prime}, \pi^{*}\left(\theta^{\prime}\right)\right)=\hat{U}\left(\theta, \theta^{\prime}, \pi^{*}(\theta)\right)>\hat{U}\left(\theta, \theta, \pi^{*}(\theta)\right)=\hat{U}\left(\theta, \varphi^{*}(\theta), \pi^{*}(\theta)\right),
$$

and (11) would be violated, a contradiction. We will see in the next section that $\pi^{S}$ is the only allocation compatible with a separating equilibrium of the informed seller game in which the belief function is non-decreasing over its entire domain.

Example 1. It is in general impossible to solve the initial value problem (18) analytically. If the buyers' types are uniformly distributed over $[0,1], \hat{\pi}(\theta, \theta)=[(1+\theta) / 2]^{2}$ for every $\theta \in[0,1]$ and, using the change of variable $\xi=\ln (1-\pi)$, it is easy to derive the following expression for the inverse $\theta^{S}$ of $\pi^{S}$ :

$$
\theta^{S}(\pi)=\frac{2(1-\lambda)(1-\pi)^{\frac{1-\lambda}{\lambda}}}{\lambda} \int_{\frac{1}{4}}^{\pi} \frac{\sqrt{x}}{(1-x)^{\frac{1}{\lambda}}} \mathrm{d} x+\left[\frac{4(1-\pi))^{\frac{1-\lambda}{\lambda}}}{3}-1\right.
$$

for all $\pi \in\left[\frac{1}{4}, 1\right]$. There is no general analytical formula for the integral in (20). When $\lambda=\frac{1}{2}$, this expression simplifies to:

$$
\theta^{S}(\pi)=2 \sqrt{\pi}+2(1-\pi)\left(\operatorname{arctanh} \frac{1}{2}-\operatorname{arctanh} \sqrt{\pi}\right)-1 .
$$

For this specification of the model, Figure 1 depicts the equilibrium probability of no trade (thick line), along with the symmetric information probability of no trade (thin line).

$$
\text { — Insert Figure } 1 \text { here - }
$$

\section{Monotone beliefs}

In this section, we study equilibria with monotone beliefs, in which the buyers' beliefs are increasing with respect to the reserve price on and off the equilibrium path. We show that equilibria with monotone beliefs are characterized by underselling relative to the buyer's beliefs and to the symmetric information benchmark, and we describe an important class of equilibria with bunching. 


\subsection{Underselling}

While the separating allocation is intuitively appealing, there is no a priori reason to rule out equilibria with bunching, in which $r^{*}$ is constant over some non-negligible set of types. It follows from Lemma 2 that, for any reserve price $r$ in the range of $r^{*}$, the seller's types that set a reserve price equal to $r$ in equilibrium form a (possibly degenerate) interval. Bunching creates discontinuities in equilibrium beliefs, allocations and reserve prices. Specifically, if one of the boundaries $\bar{\theta}$ of a bunching interval lies strictly between 0 and 1, Bayes' rule implies that $\varphi^{*}$ must be discontinuous at $\bar{\theta}$. Since $\hat{U}$ and $\hat{U}^{*}$ are continuous functions, $\pi^{*}$ and $r^{*}$ must also be discontinuous at $\bar{\theta}$.

An important remark is that, no matter the buyers' beliefs, a seller with type $\theta=0$ always wants to sell with strictly positive probability, while a seller of type $\theta=1$ never wants to sell with strictly positive probability. Hence, we have the following result.

Proposition 2. There exists no full bunching equilibrium.

A by-product of the proof of Proposition 2 is that in any equilibrium, $\pi^{*}(\theta)$ goes to 1 as $\theta$ goes to 1 . To go beyond this result, some further restrictions on equilibria are needed. This is because, as pointed out in Subsection 2.3, the informed seller game is not a standard signalling game. The key conceptual difficulty is that it is unclear from (4) whether the utility of a seller with type $\theta$ is increasing with respect to beliefs $\varphi$ for any given reserve price $r$. Indeed, the expected gains (6) from manipulating the buyers' beliefs are unambiguously increasing in $\varphi$ only if $\varphi \geq \theta$. Similarly, it is easy to check that (5) is increasing in $\varphi$ only if $z(\varphi, r) \geq \hat{z}(\theta, \theta)$ or, equivalently, if the probability of no trade $\pi(\varphi, r)$ is larger than the probability of no trade $\hat{\pi}(\theta, \theta)$ that would arise if the seller's type were common knowledge. Intuitively, an increase in buyers' beliefs unambiguously benefits the seller if private information about his type induces underselling relative to the symmetric information benchmark, and thus reduces the efficiency of trade.

While it may seem reasonable that this generalized lemon property should be satisfied in equilibrium, in line with Akerlof (1970), this is not something that we can take for granted a priori. One of the objectives of this section is to show that this intuition is indeed correct in any equilibrium with monotone beliefs, in which the belief function $\phi^{*}$ is non-decreasing over its entire domain. Our first result is that any such equilibrium exhibits underselling relative to the buyers' beliefs.

Proposition 3. In any equilibrium with monotone beliefs, the probability of no trade is strictly larger than the probability of no trade that would be optimal for the seller given the buyers' beliefs,

$$
\pi^{*}(\theta)>\hat{\pi}\left(\theta, \varphi^{*}(\theta)\right)
$$

for almost every $\theta \in[0,1]$.

The underselling property (21) is key to all the results of this section. Indeed, we shall prove that, in a sense to be made precise, it characterizes equilibria with monotone beliefs. In any equilibrium, (21) naturally holds as an equality when $\theta=1$ since, no matter the buyers' beliefs, a seller with type $\theta=1$ never wants to sale with strictly positive probability. The only other situations in which (21) may hold as an equality are when $\theta=0$, as in the separating allocation, or when $\theta$ is at the right boundary of a bunching interval. A by-product of the proof of Proposition 3 is that any discontinuity in the reserve price must occur at the boundary of a bunching interval. 
The first important consequence of Proposition 3 is that, as pointed out above, any equilibrium with monotone beliefs exhibits underselling relative to the symmetric information benchmark.

Corollary 1. In any equilibrium with monotone beliefs, the probability of no trade is strictly larger than the probability of no trade that would arise if the seller's type were common knowledge,

$$
\pi^{*}(\theta)>\hat{\pi}(\theta, \theta)
$$

for almost every $\theta \in[0,1]$.

A further consequence of Proposition 3 is relative to the probability of no trade $\pi\left(\phi^{*}(r), r\right)$ as a function of the reserve price $r$. In any equilibrium, $\pi\left(\phi^{*}(r), r\right)$ is non-decreasing for $r$ in the range of $r^{*}$, since then $\pi\left(\phi^{*}(r), r\right)=\pi^{*}\left(\left(r^{*}\right)^{-1}(r)\right)$ and $r^{*}$ and $\pi^{*}$ are non-decreasing. Our next result implies that in any equilibrium with monotone beliefs, there is no loss of generality in assuming that this property also holds for out-of-equilibrium reserve prices. The idea is that one can always modify the belief function $\phi^{*}$ off the equilibrium path on any interval of the form $\left(r^{*}\left(\bar{\theta}^{-}\right), r^{*}\left(\bar{\theta}^{+}\right)\right)$corresponding to the boundary $\bar{\theta}$ of a bunching interval so as to make a seller of type $\bar{\theta}$ indifferent between all reserve prices in this interval. This result provides the key technical tools to construct semi-bunching equilibria, see Corollary 4 and Section 5.2.

Corollary 2. For any equilibrium $\left(\phi^{*}, r^{*}\right)$ with monotone beliefs, there exists an equilibrium $\left(\phi^{* *}, r^{*}\right)$ with monotone beliefs such that the probability of no trade is non-decreasing with respect to the reserve price. Moreover, $\phi^{* *}$ can be chosen to be continuous and strictly increasing over $[0,1]$.

As pointed out in Proposition 1, the informed seller game has no full bunching equilibrium. Under monotone beliefs, this result can be refined as follows. Imagine that there exists an equilibrium in which the probability of no trade is equal to 1 on a bunching interval $(\bar{\theta}, 1]$ at the top of the seller's type distribution. Then a seller of type $\bar{\theta}$ must be indifferent between selling with strictly positive probability, by setting a reserve price $r^{*}\left(\bar{\theta}^{-}\right)$and inducing beliefs $\varphi^{*}\left(\bar{\theta}^{-}\right)$, and not selling at all. This implies that, given buyers' beliefs $\varphi^{*}\left(\bar{\theta}^{-}\right)$, he would benefit from increasing slightly the probability of no trade. However, this is incompatible with an equilibrium with monotone beliefs. Indeed, by Corollary 2, there is no loss of generality in assuming that, in such an equilibrium, the probability of no trade is non-decreasing and the belief function is strictly increasing with respect to the reserve price. Hence, a seller of type $\bar{\theta}$ could strictly improve upon his equilibrium expected payoff $\bar{\theta}$ by setting a reserve price slightly above $r^{*}\left(\bar{\theta}^{-}\right)$, as this would increase both the buyers' beliefs and the probability of no trade. We have the following result.

Corollary 3. In any equilibrium with monotone beliefs, there cannot be bunching at the top of the seller's type distribution. In particular,

$$
\pi^{*}(\theta)<1
$$

for every $\theta \in[0,1)$.

The proof of Corollary 3 shows that the no bunching at the top property (23) is a direct consequence of the underselling property (21). A key insight of this result is that in an 
equilibrium with monotone beliefs, the seller can never commit not to trade, unless of course his type is 1 . This is no longer the case if the object is sold through a monopoly broker, as we will later see. In line with the final remarks of Section 4, another important consequence of this result is that the separating allocation $\pi^{S}$ is the only allocation compatible with a separating equilibrium with monotone beliefs.

Our next result provides a characterization of all allocations consistent with an equilibrium with monotone beliefs.

Corollary 4. Consider an allocation $\left(\pi^{*}, \varphi^{*}, \hat{U}^{*}\right)$ with $\pi^{*}$ and $\varphi^{*}$ left-continuous and $\hat{U}^{*}$ continuous, such that the following conditions hold:

(i) $\varphi^{*}(\theta)=\mathrm{E}\left[\tilde{\theta} \mid \pi^{*}(\tilde{\theta})=\pi^{*}(\theta)\right]$ for all $\theta \in[0,1]$;

(ii) $\hat{U}^{*}(\theta)=\hat{U}\left(\theta, \varphi^{*}(\theta), \pi^{*}(\theta)\right)$ is convex with respect to $\theta$;

(iii) $\frac{\mathrm{d} \hat{U}^{*}}{\mathrm{~d} \theta}(\theta)=\pi^{*}(\theta)$ for almost every $\theta \in[0,1]$;

(iv) $\hat{U}^{*}(0) \geq \hat{U}(0,0, \hat{\pi}(0,0))$;

(v) $\pi^{*}(\theta)>\hat{\pi}\left(\theta, \varphi^{*}(\theta)\right)$ for almost every $\theta \in[0,1]$.

Then there exists an equilibrium with monotone beliefs that supports this allocation.

This characterization is stated in terms of the auxiliary game in which the seller directly chooses the probability of no trade $\pi$ and has expected utility $\hat{U}(\theta, \varphi, \pi)$. Note that conditions (i)-(iv) are standard implementability conditions for this game. By contrast, the additional restriction (v) coincides with the underselling property (21) and is the key to the result. Indeed, what Corollary 4 shows is that this condition is sufficient to transform an equilibrium of the standard auxiliary game into an equilibrium of the informed seller game.

Before we turn to the analysis of semi-bunching equilibria, we show that, if the requirement of monotone beliefs is dropped, then it is possible to construct equilibria of the informed seller game that satisfy neither the underselling property (21), nor the no bunching at the top property (23). Specifically, we have the following result.

Proposition 4. Suppose that for some $\bar{\theta} \in(1-\lambda, 1)$, there exists a pair $(\bar{\varphi}, \bar{\pi}) \in(0, \bar{\theta}) \times(0,1)$ such that the following conditions hold:

(i) $\hat{U}(\bar{\theta}, \bar{\varphi}, \bar{\pi})=\bar{\theta}$;

(ii) $\hat{U}(0, \bar{\varphi}, \bar{\pi}) \geq \hat{U}(0,0, \hat{\pi}(0,0))$.

Then there exists a seller's type distribution $G$ such that the informed seller game has an equilibrium with non-monotone beliefs in which:

$$
\pi^{*}(\theta)= \begin{cases}\bar{\pi} & \text { if } \quad \theta \leq \bar{\theta}, \\ 1 & \text { if } \quad \theta>\bar{\theta} .\end{cases}
$$

This equilibrium exhibits overselling relative to buyer's beliefs at $\bar{\theta}, \bar{\pi}<\hat{\pi}(\bar{\theta}, \bar{\varphi})$, and bunching at the top of the seller's type distribution.

In the equilibrium constructed in Proposition 4, only two reserve prices can occur on the equilibrium path, namely $\bar{r}=\lambda \bar{\varphi}+(1-\lambda)\left(F^{2}\right)^{-1}(\bar{\pi})$ and 1 . The key to the proof is that 
whenever $\bar{\theta}>1-\lambda, \hat{U}(\bar{\theta}, 0, \pi)$ is a strictly increasing function of $\pi$ over the whole interval $[0,1]$. This ensures that, by imposing out-of-equilibrium beliefs to be 0 on $(\bar{r}, 1)$, a seller with type $\theta \geq \bar{\theta}$ does not want to deviate to a reserve price $r \in(\bar{r}, 1)$. This implies in turn that a seller with type $\theta<\bar{\theta}$ does not want to deviate to such a reserve price either, as this would increase the probability of no trade and decrease beliefs. Last, it should be noted that any allocation $\left(\pi^{*}, \varphi^{*}, \hat{U}^{*}\right)$ consistent with such an equilibrium automatically satisfies the implementability conditions (i)-(iv) of the auxiliary game, as given in Corollary 4. This illustrates the fact that the the underselling condition (v) provides a tight characterization of allocations consistent with equilibria with monotone beliefs.

Example 2. Conditions (i)-(ii) of Proposition 4 can be readily verified in an example. Suppose that the buyers' types are uniformly distributed over $[0,1]$ as in Example 1, and let $\lambda=\frac{1}{2}$ and $\bar{\pi}=\frac{1}{4}=\hat{\pi}(0,0)$. Then if we find some $\bar{\theta} \in\left(\frac{1}{2}, 1\right)$ and $\bar{\varphi} \in(0, \bar{\theta})$ that satisfy condition (i), condition (ii) will be automatically satisfied. Condition (i) can be rewritten as:

$$
\frac{\bar{\theta}}{4}+\frac{3 \bar{\varphi}}{8}+\frac{1}{2} \int_{\frac{1}{2}}^{1} 2(2 \zeta-1) \zeta \mathrm{d} \zeta=\bar{\theta},
$$

or $\bar{\varphi}=2 \bar{\theta}-\frac{5}{9}$. It is immediate to check that, for any $\bar{\theta} \in\left(\frac{1}{2}, \frac{5}{9}\right), \bar{\varphi}<\bar{\theta}$, and thus the parameter restrictions of Proposition 4 are satisfied. Note that the equilibrium allocation thus constructed exhibits overselling relative the symmetric information benchmark for all $\theta \in(0, \bar{\theta}]$, and overselling relative to the buyers' beliefs for all $\theta \in\left(\frac{\bar{\varphi}}{2}, \bar{\theta}\right]$. On $[0, \bar{\theta}]$, the equilibrium reserve price is $\bar{r}=\bar{\theta}-\frac{1}{36}$, so that a seller with type $\theta \in\left(\bar{\theta}-\frac{1}{36}, \bar{\theta}\right]$ sets a reserve price below his own valuation for the object.

\subsection{Some semi-bunching equilibria}

We now turn to the discussion of semi-bunching equilibria. A full characterization would be out of scope, and we focus instead on a particular type of equilibria leading to the maximal degree of bunching consistent with monotone beliefs. From Corollary 3, we know that it is not possible to have bunching at the top of the seller's type distribution in an equilibrium with monotone beliefs. We thus concentrate on equilibria of the following form:

(a) Seller's types $\theta \in[0, \bar{\theta}]$ announce a reserve price $\bar{r}$;

(b) Seller's types $\theta \in(\bar{\theta}, 1]$ announce reserve prices $r^{*}(\theta)$, where $r^{*}$ is strictly increasing.

For each $\theta \in[0,1]$, define $\bar{\varphi}(\theta)=\mathrm{E}[\tilde{\theta} \mid \tilde{\theta} \leq \theta]$. Then, in any equilibrium satisfying (a)-(b), the equilibrium belief function must be:

$$
\varphi^{*}(\theta)=\left\{\begin{array}{cc}
\bar{\varphi}(\bar{\theta}) & \text { if } \quad \theta \leq \bar{\theta}, \\
\theta & \text { if } \quad \theta>\bar{\theta} .
\end{array}\right.
$$

Let $\bar{\pi}$ be the equilibrium probability of no trade given the reserve price $\bar{r}$. A straightforward adjustment of the analysis of Section 4 and Corollary 4 then shows that the equilibrium conditions reduce to:

$$
\begin{aligned}
\hat{U}(\bar{\theta}, \bar{\varphi}(\bar{\theta}), \bar{\pi}) & =\hat{U}\left(\bar{\theta}, \bar{\theta}, \pi^{*}\left(\bar{\theta}^{+}\right)\right), \\
\hat{U}(0, \bar{\varphi}(\bar{\theta}), \bar{\pi}) & \geq \hat{U}(0,0, \hat{\pi}(0,0)), \\
\bar{\pi} & \geq \hat{\pi}(\bar{\theta}, \bar{\varphi}(\bar{\theta})),
\end{aligned}
$$


together with the requirement that $\pi^{*}$ satisfies the differential equation (17) over $(\bar{\theta}, 1]$. Such an equilibrium is fully characterized by $(\bar{\theta}, \bar{\pi})$, and will be referred to as an equilibrium with bunching below $\bar{\theta}$ at $\bar{\pi}$. In particular, provided that $\pi^{*}\left(\bar{\theta}^{+}\right)<1$, the differential equation (17) has a strictly increasing solution over $(\bar{\theta}, 1]$ starting at $\left(\bar{\theta}, \pi^{*}\left(\bar{\theta}^{+}\right)\right)$, and this solution is unique. ${ }^{6}$ Thus a sufficient condition for a pair $(\bar{\theta}, \bar{\pi})$ to be consistent with an equilibrium of this type is that (25) admits a solution $\pi^{*}\left(\bar{\theta}^{+}\right)<1$, along with (26) and (27).

A key observation is that $\hat{U}(\bar{\theta}, \bar{\varphi}(\bar{\theta}), \bar{\pi})$ is a decreasing function of $\bar{\pi}$ over the relevant range defined by (27). This implies the following result.

Lemma 4. For any equilibrium with bunching below $\bar{\theta}$ at $\bar{\pi}$, there exists an equilibrium with bunching below $\bar{\theta}$ at $\hat{\pi}(\bar{\theta}, \bar{\varphi}(\bar{\theta}))$, referred to as a $[0, \bar{\theta}]$-equilibrium, which leads to a higher probability of trade and a higher profit for all seller's types.

$[0, \bar{\theta}]$-equilibria are thus the most efficient equilibria that satisfy (a)-(b). For this reason, we shall focus on them. Existence conditions then reduce to:

$$
\begin{aligned}
& \hat{U}(\bar{\theta}, \bar{\varphi}(\bar{\theta}), \hat{\pi}(\bar{\theta}, \bar{\varphi}(\bar{\theta})))>\bar{\theta} \\
& \hat{U}(0, \bar{\varphi}(\bar{\theta}), \hat{\pi}(\bar{\theta}, \bar{\varphi}(\bar{\theta}))) \geq \hat{U}(0,0, \hat{\pi}(0,0)) .
\end{aligned}
$$

Notice that since $\bar{\varphi}(\bar{\theta})<\bar{\varphi}(1)<1$, a seller with a large type would refuse to sell for these beliefs, that is $\hat{U}(\bar{\theta}, \bar{\varphi}(\bar{\theta}), \hat{\pi}(\bar{\theta}, \bar{\varphi}(\bar{\theta})))=\bar{\theta}$ for $\bar{\theta}$ large but smaller than 1 . Thus there is an upper bound on the size of the bunching interval $[0, \bar{\theta}]$. One has the following result.

Proposition 5. A $[0, \bar{\theta}]$-equilibrium always exists for $\bar{\theta}$ small enough, and never exists for $\bar{\theta}$ large enough.

Note that the bounds on $\bar{\theta}$ below which a $[0, \bar{\theta}]$-equilibrium necessarily exists and above which a $[0, \bar{\theta}]$-equilibrium never exists crucially depend on the distribution of the seller's type.

\section{Trading with a monopoly broker}

In this section, we consider the situation where a monopoly broker, uninformed about the seller's and the buyers' types, designs the trading mechanism, as in Myerson and Satterthwaite (1983). We first characterize the mechanism that maximizes the broker's expected profit. Next, we discuss the implementation of this mechanism.

\subsection{The monopoly broker allocation}

By the Revelation Principle, there is no loss in generality in focusing on direct revelation mechanisms $\{p(\mathcal{T}), t(\mathcal{T})\}_{\mathcal{T} \in[0,1]^{3}}$ for the seller and $\left\{p^{i}(\mathcal{T}), t^{i}(\mathcal{T})\right\}_{\mathcal{T} \in[0,1]^{3}}, i=1,2$, for the buyers, where the $p$ component refers to the probability that the agent receives the good, and the $t$ component refers to the expected transfer to the broker, both conditional on the realization of the vector of types $\tilde{\mathcal{T}}=\left(\tilde{\theta}, \tilde{\zeta}^{1}, \tilde{\zeta}^{2}\right)$. The interim utility of the seller is:

$$
U^{B}(\theta)=\mathrm{E}[\tilde{\theta} p(\tilde{\mathcal{T}})-t(\tilde{\mathcal{T}}) \mid \tilde{\theta}=\theta]
$$

\footnotetext{
${ }^{6}$ Specifically, it can be shown along the lines of Proposition 1 that the differential equation (17) has an infinite number of strictly increasing solutions over $(\bar{\theta}, 1]$, all converging to 1 when $\theta$ goes to 1 . These solutions are ranked and characterized by an initial value $\pi \in[\hat{\pi}(\bar{\theta}, \bar{\theta}), 1)$. Provided that $\pi^{*}\left(\bar{\theta}^{+}\right)<1$, this condition is satisfied in the present case since, from $(27)$ and the fact that $\hat{\pi}(\bar{\theta}, \varphi)$ is strictly decreasing with respect to $\varphi$, one has $\pi^{*}\left(\bar{\theta}^{+}\right)>\bar{\pi}>\hat{\pi}(\bar{\theta}, \bar{\theta})$.
} 
while that of buyer $i=1,2$, is:

$$
U^{B, i}\left(\zeta^{i}\right)=\mathrm{E}\left[\tilde{\theta}^{i} p^{i}(\tilde{\mathcal{T}})-t^{i}(\tilde{\mathcal{T}}) \mid \tilde{\zeta}^{i}=\zeta^{i}\right] .
$$

The incentive compatibility conditions imply that the mappings $U^{B}$ and $U^{B, i}, i=1,2$, are almost everywhere differentiable, and their derivatives are given by the usual envelope conditions. The broker's objective is to maximize his expected profit:

$$
\mathrm{E}\left[\tilde{\theta} p(\tilde{\mathcal{T}})+\sum_{i=1}^{2} \tilde{\theta}^{i} p^{i}(\tilde{\mathcal{T}})-U^{B}(\tilde{\theta})-\sum_{n=1}^{2} U^{B, i}\left(\tilde{\zeta}^{i}\right)\right],
$$

subject to the incentive compatibility and individual rationality constraints for the seller,

$$
\begin{aligned}
\frac{\mathrm{d} U^{B}}{\mathrm{~d} \theta}(\theta) & =\mathrm{E}[p(\tilde{\mathcal{T}}) \mid \tilde{\theta}=\theta], \\
U^{B}(\theta) & \geq \theta,
\end{aligned}
$$

the incentive compatibility and individual rationality constraints for buyer $i=1,2$,

$$
\begin{aligned}
\frac{\mathrm{d} U^{B, i}}{\mathrm{~d} \zeta^{i}}\left(\zeta^{i}\right) & =(1-\lambda) \mathrm{E}\left[p^{i}(\tilde{\mathcal{T}}) \mid \tilde{\zeta}^{i}=\zeta^{i}\right] \\
U^{B, i}(0) & =0,
\end{aligned}
$$

and the feasibility constraint,

$$
p(\mathcal{T})+\sum_{n=1}^{2} p^{i}(\mathcal{T}) \leq 1,
$$

for every $\mathcal{T}=\left(\theta, \zeta^{1}, \zeta^{2}\right) \in[0,1]^{3}$. Note that the feasibility constraint will hold as an equality in an optimal mechanism. Otherwise, the object would have to be destroyed. Since $\frac{\mathrm{d} U^{B}}{\mathrm{~d} \theta} \leq 1$, the type-dependent participation constraint for the seller can be replaced by $U^{B}(1)=1$. One has then the following result.

Lemma 5. In the profit maximizing broker's mechanism, the object is traded if and only if:

$$
\max _{i \in\{1,2\}}\left\{\tilde{\zeta}^{i}-\frac{1-F\left(\tilde{\zeta}^{i}\right)}{f\left(\tilde{\zeta}^{i}\right)}\right\} \geq \tilde{\theta}+\frac{G(\tilde{\theta})}{(1-\lambda) g(\tilde{\theta})} .
$$

The monopoly broker allocation is then completely characterized by (30). In particular, let $\theta^{B}<1$ be the solution to:

$$
\theta+\frac{G(\theta)}{(1-\lambda) g(\theta)}=1
$$

Then, in the monopoly broker allocation, the object is never traded if the seller's type is larger than $\theta^{B}$. The intuition is that high quality objects are not put for sale in order to allow the broker to better extract rents from sellers with lower quality objects. Specifically, in order to raise his own revenue, the broker must prevent the seller to overstate his type. This in turn calls for larger distortions when the sellers claims to have a high type. For every $\theta<\theta^{B}$, define $z^{B}(\theta)$ as the unique solution to:

$$
z^{B}(\theta)-\frac{1-F\left(z^{B}(\theta)\right)}{f\left(z^{B}(\theta)\right)}=\theta+\frac{G(\theta)}{(1-\lambda) g(\theta)}
$$


and for $\theta \geq \theta^{B}$, let $z^{B}(\theta)=1$. Then, given the seller's type $\theta$, the probability of no trade in the monopoly broker allocation is $\pi^{B}(\theta)=F\left(z^{B}(\theta)\right)^{2}$. The monotone hazard rate property for $F$ and $G$ implies that $z^{B}(\theta)$ and therefore $\pi^{B}(\theta)$ are strictly increasing with respect to $\theta$. Note that from (10) and $(31), \pi^{B}(\theta) \geq \hat{\pi}(\theta, \theta)$, with equality at 0 and 1 only. It will be assumed that $z^{B}(\theta)$ is a differentiable function of $\theta$, with a uniformly bounded derivative over $[0,1]$.

\subsection{Implementation}

A natural question is whether the monopoly broker allocation can be implemented by a simple mechanism. For every $\theta \in[0,1]$, define a reserve price as:

$$
r^{B}(\theta)=\lambda \theta+(1-\lambda) z^{B}(\theta) .
$$

Then it is clear that if the buyers know the seller's type $\theta$, a second-price sealed bid auction with reserve price $r^{B}(\theta)$ implements the monopoly broker allocation. Moreover, since $r^{B}(\theta)$ is strictly increasing with respect to $\theta$, it follows that if the seller is induced to choose a public reserve price $r^{B}(\theta)$ when his type is $\theta$ and a second-price sealed bid auction is organized, the choice of the reserve price will reveal the seller's type to the buyers, and the monopoly broker allocation will be implemented. This leads to the following simple mechanism. Define a mapping $\mathcal{F}^{B}$ on the range of $r^{B}$ by the relation:

$$
\mathcal{F}^{B}\left(r^{B}(\theta)\right)=\hat{U}\left(\theta, \theta, \pi^{B}(\theta)\right)-U^{B}(\theta),
$$

and consider the following mechanism:

(i) The broker sets a menu $\mathcal{F}^{B}$ of transaction fees contingent on the reserve price;

(ii) The seller publicly announces a reserve price $r$ and pays the transaction fee $\mathcal{F}^{B}(r)$;

(iii) The buyers observe the menu $\mathcal{F}^{B}$ and the reserve price $r$, and engage in a second-price sealed bid auction with reserve price $r$ in which no fees are paid to the broker.

Note that the menu $\mathcal{F}^{B}$ can take the form of a fixed registration fee to be paid by the seller in order to participate to the mechanism, but could also obtain with an ex-post transaction fee. It is immediate to verify that incentive compatibility of the monopoly broker allocation implies that there exists a separating equilibrium of the game induced by this mechanism in which a seller of type $\theta$ chooses the reserve price $r^{B}(\theta)$ and receives interim expected utility $U^{B}(\theta)$, buyer $i=1,2$, receives interim expected utility $U^{B, i}\left(\zeta^{i}\right)$ whenever his type is $\zeta^{i}$, and the object is not traded with probability $\pi^{B}(\theta)$.

An important consequence of this result is that it shows that the comparison between the monopoly broker solution and the informed seller allocation is not driven by the fact that the broker has access to a larger set of trading mechanisms. Indeed, the solution would be the same if the broker were restricted to use only second-price sealed bid auctions with an announced reserve price. Instead, the comparison is driven by the fact that the broker can tie the seller's fee to the reserve price and use this contingent transfer as a screening device.

A key assumption is that the monopoly intermediary is committed to the mechanism. In particular, if the reserve price chosen by the seller were only observed by the intermediary who would then transmit it to buyers, there might be incentives for the broker to renegotiate with the seller. In such a context, there must be some transaction cost or reputation mechanism that prevents renegotiation. To the extent that reputation mechanisms may create barriers to entry, this may provide an ex-post rationale for the market power assumed in our paper. 


\section{Informed seller versus monopoly broker}

The issue addressed in this section is whether, despite the distortion due to market power, the monopoly broker can generate a more efficient allocation than a decentralized market structure in which the seller chooses freely the reserve price. We first show that, at the interim stage, the comparison between these two types of allocation mechanisms is ambiguous. Specifically, compared with the allocations that may arise if the seller directly trades with the buyers, the broker tends to increase the probability of trade for low values of the seller's type, but to reduce it for high values. We then turn to ex-ante efficiency comparisons, focusing on the unique separating allocation characterized in Section 4. Our main result is that whenever the seller's type distribution is enough concentrated, the ex-ante probability of trade and the ex-ante surplus may be larger with a monopoly broker than when the seller directly trades with the buyers.

\subsection{Interim efficiency comparisons}

In this section, we focus on interim efficiency comparisons, conditional on the seller's type. The simplest type of comparison between the different allocation mechanisms considered thus far consists to determine under which circumstances the object can be traded with strictly positive probability. We have seen that in the monopoly broker allocation, the object is never traded if the seller's type is above $\theta^{B}<1$. By contrast, Corollary 3 implies that in any equilibrium of the informed seller game with monotone beliefs, the probability of trade is strictly positive, unless the seller's type is equal to 1 . Thus the monopoly broker allocation is less efficient at the top of the seller's type distribution than any equilibrium allocation compatible with monotone beliefs. Note also that sellers with types above $\theta^{B}$ would be better off selling the good themselves than through a monopoly broker.

By contrast, consider what happens for seller's types close to 0. Two cases must be distinguished. First, if there is bunching at 0 in an equilibrium with monotone beliefs, then by Corollary $1, \pi^{*}\left(0^{+}\right)>\hat{\pi}(0,0)$. Since $\pi^{B}(0)=\hat{\pi}(0,0)$ and $\pi^{B}$ is continuous, it follows that $\pi^{B}(\theta)<\pi^{*}(\theta)$ for $\theta>0$ close enough to 0 . Next, if the equilibrium is separating at 0 , then it is not difficult to prove along the lines of Proposition 1 that $\pi^{*}$ must coincide with $\pi^{S}$ in a neighborhood of 0 . Since $\pi^{B}$ is differentiable at 0 , in contrast with $\pi^{S}$, and $\pi^{B}(0)=\pi^{S}(0)=\hat{\pi}(0,0)$, it follows that $\pi^{B}(\theta)<\pi^{S}(\theta)$ for $\theta>0$ close enough to 0 . Thus the monopoly broker allocation is more efficient at the bottom of the seller's type distribution than any equilibrium allocation compatible with monotone beliefs.

Remark. It should be noted that this result depends crucially on the assumption of common values, that is $\lambda>0$. If $\lambda=0$, we are in the standard private values situation. All the characterizations obtained so far generalize to this limit case. In particular, one has $\pi^{S}(\theta)=$ $\hat{\pi}(\theta, \theta)<\pi^{B}(\theta)$ for each $\theta \in(0,1)$, and the monopoly broker increases the probability of no trade in two ways. First, the seller is prevented from trading if his type is larger than $\theta^{B}<1$. Second, the reserve price set by the broker is higher than if the seller sells the object himself. Thus, in the private values case, having a monopoly broker is always less efficient than letting the seller directly trade with the buyers, and the revenue to the seller is also less.

Example 3. For the specification of the model given in Example 1, Figure 2 depicts the probability of no trade in the separating equilibrium (thick line), along with the probability of no trade in the monopoly broker allocation (thin line), under the assumption that the seller's type is uniformly distributed over $[0,1]$. 


\subsection{Ex-ante efficiency comparison}

We now turn to ex-ante efficiency comparisons. Such comparisons are difficult to perform for equilibria that exhibit bunching, as they depend crucially on the seller's type distribution. By contrast, the separating equilibrium allocation characterized in Section 4 depends only on the support of the seller's type, and is therefore easier to handle.

We first focus on the ex-ante probability of trade. Our objective is to compare the ex-ante probability of trade in the separating equilibrium allocation of the informed seller game, $\mathcal{P}^{S}$, with the ex-ante probability of trade in the monopoly broker mechanism, $\mathcal{P}^{B}$. To simplify notation, let $\hat{z}(\theta)=\hat{z}(\theta, \theta)$ for $\theta \in[0,1]$, where $\hat{z}(\theta, \theta)$ is as before the unique solution to (10), and extend $\hat{z}$ by continuity by setting $\hat{z}(\theta)=1$ for $\theta \in[1, \infty)$. Under our assumptions, $\hat{z}$ has a uniformly bounded derivative over $\mathbb{R}_{+}$. One then has:

$$
\begin{aligned}
\mathcal{P}^{S} & =1-\int_{0}^{1} F\left(z^{S}(\theta)\right)^{2} g(\theta) \mathrm{d} \theta, \\
\mathcal{P}^{B} & =1-\int_{0}^{1} F\left(\hat{z}\left(\theta+\frac{G(\theta)}{(1-\lambda) g(\theta)}\right)\right)^{2} g(\theta) \mathrm{d} \theta .
\end{aligned}
$$

We shall now prove that $\mathcal{P}^{B}>\mathcal{P}^{S}$ whenever $G$ is sufficiently concentrated around some value of the seller's type. Formally, for any $\varepsilon \in(0,1)$, we will say that $G$ is $\varepsilon$-concentrated at $\theta_{0} \in(\varepsilon, 1-\varepsilon)$ if:

$$
G\left(\theta_{0}+\varepsilon\right)-G\left(\theta_{0}-\varepsilon\right) \geq 1-\varepsilon .
$$

One has then the following result.

Proposition 6. For every $\theta_{0} \in(0,1)$, there exists $\varepsilon_{0} \in(0,1)$ such that for every $\varepsilon \in\left(0, \varepsilon_{0}\right)$, $\mathcal{P}^{B}>\mathcal{P}^{S}$ whenever $G$ is $\varepsilon$-concentrated at $\theta_{0}$.

The intuition of this result is that, as $G$ becomes increasingly concentrated at $\theta_{0}$, the ex-ante probability of trade $\mathcal{P}^{B}$ in the monopoly broker allocation becomes close to the symmetric information probability of trade, $1-\int_{0}^{1} F(\hat{z}(\theta))^{2} g(\theta) \mathrm{d} \theta$. It is interesting to note that there is a discontinuity in terms of ex-ante probability of trade as $\lambda$ goes to 0 , that is as one becomes close to a pure private values situation. When $\lambda=0$, a monopoly broker always reduce the ex-post amount of trade compared to the case where the owner of the good sells the good himself, by charging a higher reserve price and by excluding high types of the seller from trade. However, as soon as $\lambda>0$, there exist distributions of the seller's type such that the ex-ante probability of trade is higher with the monopoly broker than in the separating equilibrium allocation of the informed seller game.

Example 4. For the specification of the model given in Example 1, Figure 3 depicts the behavior of the probability of no trade in the monopoly broker allocation as the seller's type distribution becomes more concentrated around $\theta_{0}=\frac{1}{2} \cdot{ }^{7}$ As this happens, the probability of no trade in the monopoly broker allocation comes closer to the symmetric information probability of no trade over the interval $\left[0, \frac{1}{2}\right]$, while the probability of no sale in the separating equilibrium is unaffected.

\section{— Insert Figure 3 here -}

\footnotetext{
${ }^{7}$ The two curves for the monopoly broker allocation depicted on Figure 3 correspond respectively to a uniform distribution and to a Beta $(15,15)$ distribution for the seller's type.
} 
We now turn to the ex-ante surplus. Our objective is to compare the ex-ante total surplus in the separating equilibrium allocation of the informed seller game, $\mathcal{S}^{S}$, with the ex-ante total surplus in the monopoly broker mechanism, $\mathcal{S}^{B}$. Specifically, one has:

$$
\begin{aligned}
& \mathcal{S}^{S}=\mathrm{E}[\tilde{\theta}]+2(1-\lambda) \int_{0}^{1} \int_{z^{S}(\theta)}^{1} F(\zeta)(\zeta-\theta) f(\zeta) \mathrm{d} \zeta g(\theta) \mathrm{d} \theta \\
& \mathcal{S}^{B}=\mathrm{E}[\tilde{\theta}]+2(1-\lambda) \int_{0}^{1} \int_{\hat{z}\left(\theta+\frac{G(\theta)}{(1-\lambda) g(\theta)}\right)}^{1} F(\zeta)(\zeta-\theta) f(\zeta) \mathrm{d} \zeta g(\theta) \mathrm{d} \theta .
\end{aligned}
$$

One has then the following result.

Proposition 7. For every $\theta_{0} \in(0,1)$, there exists $\varepsilon_{0} \in(0,1)$ such that for every $\varepsilon \in\left(0, \varepsilon_{0}\right)$, $\mathcal{S}^{B}>\mathcal{S}^{S}$ whenever $G$ is $\varepsilon$-concentrated at $\theta_{0}$.

As for the ex-ante probability of trade, the intuition of this result is that when there is little asymmetric information about the seller's type, a screening mechanism is more efficient than the separating equilibrium of the signalling game, which does not depend on the distribution of the seller's type.

Key to the above results is the fact that the ex-ante probability of no trade induced by the monopoly broker is close to $\hat{\pi}\left(\theta_{0}, \theta_{0}\right)$ whenever the distribution is concentrated at $\theta_{0}$, while this is not the case for the separating equilibrium. One may then wonder to which extent the results extend to other equilibria of the informed seller game. Indeed, it is well-known that the separating equilibrium may perform poorly on efficiency (Laffont and Maskin, 1990, Glosten, 1994), and that pooling equilibria may perform better when the distribution is concentrated (Mailath, Okuno-Fujira and Postlewaite, 1993)..$^{8}$

While it is difficult to answer this question for arbitrary semi-bunching equilibria, the case of $[0, \bar{\theta}]$-equilibria as defined in Subsection 5.2 is relatively easy to address. If the seller's type distribution $G$ is $\varepsilon$-concentrated at $\theta_{0}$ for $\varepsilon$ close to 0 , and $\bar{\theta}$ is above but close to $\theta_{0}$, then the expected probability of no trade is close to $\hat{\pi}\left(\theta_{0}, \theta_{0}\right)$ since $\bar{\varphi}(\bar{\theta})$ is close to $\theta_{0}$. The equilibrium allocation is then close to the efficient allocation at $\theta_{0}$. In this case, there is no obvious way to compare the monopoly broker allocation and the $[0, \bar{\theta}]$-equilibrium allocation in an ex-ante sense. However, the above reasoning requires that $\hat{U}\left(0, \theta_{0}, \hat{\pi}\left(\theta_{0}, \theta_{0}\right)\right)$ be greater than $\hat{U}(0,0, \hat{\pi}(0,0))$ for such a $[0, \bar{\theta}]$-equilibrium to exist whenever the distribution $G$ becomes increasingly concentrated at $\theta_{0}$. This condition is in turn only satisfied if $\theta_{0}$ is below some threshold. When $\theta_{0}$ is large, this condition is violated and a $[0, \bar{\theta}]$-equilibrium does not exist for $\bar{\theta} \geq \theta_{0}$ and a seller's type distribution that is sufficiently concentrated at $\theta_{0}$. By Proposition 5, $[0, \bar{\theta}]$-equilibria will typically exists for $\bar{\theta}<\theta_{0}$ close enough to 0 , but these equilibria will be separating at $\theta_{0}$, and the same reasoning as in Propositions 6 and 7 implies that they imply a lower ex-ante probability of trade and a lower ex-ante surplus than the monopoly broker allocation.

\section{Conclusion}

Our paper proposes a solution to the problem of the design of auctions by informed sellers. In doing so, it shows that the standard result that the lemon problem leads to an inefficient reduction in the volume of trade still holds in an auction context. The conclusion relies only

\footnotetext{
${ }^{8}$ We thank an anonymous referee for pointing this point to us and for providing references.
} 
on the monotonicity of beliefs with respect to the reserve price, which seems a reasonable assumption since it holds on the equilibrium path.

Still, we restricted attention to mechanisms where the seller chooses a selling mechanism and is prevented from being an active participant of the mechanism once this choice is made. In this case we can focus on second-price sealed bid auctions with a reserve price with no loss of generality (see footnote 2 and Jullien and Mariotti, 2003). It would be useful to determine whether allowing for more sophisticated mechanisms would lead to the same conclusions.

A second contribution of the paper is to solve for the optimal mechanism of a monopoly broker and to compare the two extreme situations of a decentralized market and of monopoly intermediation. We have shown that monopoly intermediation may dominate the separating equilibrium of the decentralized market structure in terms of expected probability of trade and expected surplus, despite the natural tendency of the monopoly to restrict trade.

This points to the fact that in a world with imperfect information, intermediation may generate some value even if it does not involve any expertise, certification, or value added services. Here the value is generated only by the ability of the intermediary to affect the design of the incentive schemes. Obviously this ability is related to the intermediary market power in our model. Thus our analysis implicitly assume so far the presence of some barrier to entry. A natural extension would be to allow for competition between brokers who design trading mechanisms and compete in order to attract buyers and sellers. Because of the twosided nature of such a market (see Rochet and Tirole, 2003), the outcome of this competitive game is unclear and should be investigated. 


\section{Appendix A}

Proof of Lemma 1. It follows from (8) that $\hat{U}(\theta, \varphi, \pi)=\hat{U}\left(\theta^{\prime}, \varphi, \pi\right)+\left(\theta-\theta^{\prime}\right) \pi$ for any $\left(\theta, \theta^{\prime}, \varphi, \pi\right) \in[0,1]^{4}$. Thus, by $(11)$ :

$$
\hat{U}^{*}(\theta) \geq \hat{U}\left(\theta, \varphi^{*}\left(\theta^{\prime}\right), \pi^{*}\left(\theta^{\prime}\right)\right)=\hat{U}^{*}\left(\theta^{\prime}\right)+\left(\theta-\theta^{\prime}\right) \pi^{*}\left(\theta^{\prime}\right),
$$

and similarly:

$$
\hat{U}^{*}\left(\theta^{\prime}\right) \geq \hat{U}\left(\theta^{\prime}, \varphi^{*}(\theta), \pi^{*}(\theta)\right)=\hat{U}^{*}(\theta)+\left(\theta^{\prime}-\theta\right) \pi^{*}(\theta) .
$$

Combining these two inequalities yields:

$$
\left(\theta-\theta^{\prime}\right) \pi^{*}(\theta) \geq \hat{U}^{*}(\theta)-\hat{U}^{*}\left(\theta^{\prime}\right) \geq\left(\theta-\theta^{\prime}\right) \pi^{*}\left(\theta^{\prime}\right)
$$

from which the result follows. Note that (32) implies that $\hat{U}^{*}$ is Lipschitz, and therefore almost everywhere differentiable on $[0,1]$.

Proof of Lemma 2. Let $\theta>\theta^{\prime}$ be such that $\pi^{*}(\theta)<1$, and suppose by way of contradiction that $r^{*}(\theta)<r^{*}\left(\theta^{\prime}\right)$. By Lemma $1, \pi^{*}(\theta) \geq \pi^{*}\left(\theta^{\prime}\right)$ and thus $z^{*}(\theta) \geq z^{*}\left(\theta^{\prime}\right)$. Hence, by (2) and the definition of $z^{*}$, it must be that $\varphi^{*}(\theta)<\varphi^{*}\left(\theta^{\prime}\right)$, or:

$$
\mathrm{E}\left[\tilde{\theta} \mid r^{*}(\tilde{\theta})=r^{*}(\theta)\right]<\mathrm{E}\left[\tilde{\theta} \mid r^{*}(\tilde{\theta})=r^{*}\left(\theta^{\prime}\right)\right]
$$

Two cases must be distinguished. Either there exists $\theta^{\prime \prime}<\theta^{\prime}$ such that $r^{*}\left(\theta^{\prime \prime}\right)=r^{*}(\theta)$, and thus $\pi^{*}\left(\theta^{\prime \prime}\right)=\pi^{*}(\theta)$. Since $\theta^{\prime \prime}<\theta^{\prime}$, it follows from Lemma 1 that $\pi^{*}(\theta) \leq \pi^{*}\left(\theta^{\prime}\right)$ and therefore $\pi^{*}(\theta)=\pi^{*}\left(\theta^{\prime}\right)$. Or there exists $\theta^{\prime \prime}>\theta$ such that $r^{*}\left(\theta^{\prime \prime}\right)=r^{*}\left(\theta^{\prime}\right)$, and thus $\pi^{*}\left(\theta^{\prime \prime}\right)=\pi^{*}\left(\theta^{\prime}\right)$. Since $\theta^{\prime \prime}>\theta$, it follows from Lemma 1 that $\pi^{*}(\theta) \leq \pi^{*}\left(\theta^{\prime}\right)$ and therefore $\pi^{*}(\theta)=\pi^{*}\left(\theta^{\prime}\right)$ again. But then, since $\varphi^{*}\left(\theta^{\prime}\right)>\varphi^{*}(\theta)$ and $\hat{U}\left(\theta, \varphi, \pi^{*}(\theta)\right)$ is strictly increasing with respect to $\varphi$ as $\pi^{*}(\theta)<1$ by assumption, one has:

$$
\hat{U}\left(\theta, \varphi^{*}\left(\theta^{\prime}\right), \pi^{*}\left(\theta^{\prime}\right)\right)=\hat{U}\left(\theta, \varphi^{*}\left(\theta^{\prime}\right), \pi^{*}(\theta)\right)>\hat{U}\left(\theta, \varphi^{*}(\theta), \pi^{*}(\theta)\right),
$$

which violates the incentive compatibility condition (11), a contradiction. Therefore $r^{*}$ is non-decreasing over $\left\{\theta \in[0,1] \mid \pi^{*}(\theta)<1\right\}$, as claimed. It follows in particular that for any $r>r^{\prime}$ in $r^{*}\left(\left\{\theta \in[0,1] \mid \pi^{*}(\theta)<1\right\}\right)$, one has:

$$
\mathrm{E}\left[\tilde{\theta} \mid r^{*}(\tilde{\theta})=r, \pi(\tilde{\theta})<1\right] \geq \mathrm{E}\left[\tilde{\theta} \mid r^{*}(\tilde{\theta})=r^{\prime}, \pi(\tilde{\theta})<1\right] .
$$

Now suppose that:

$$
\phi^{*}(r)=\mathrm{E}\left[\tilde{\theta} \mid r^{*}(\tilde{\theta})=r\right]<\mathrm{E}\left[\tilde{\theta} \mid r^{*}(\tilde{\theta})=r^{\prime}\right]=\phi^{*}\left(r^{\prime}\right) .
$$

Then, since $\left\{\theta \in[0,1] \mid \pi^{*}(\theta)=1\right\}$ is of the form $\left[\theta_{0}, 1\right]$ by Lemma $1,(33)-(34)$ imply that there exists a positive mass of types $\theta$ such that $r^{*}(\theta)=r^{\prime}$ and $\pi^{*}(\theta)=1$. Hence, by Bayes' rule, one must have $r^{\prime} \notin r^{*}\left(\left\{\theta \in[0,1] \mid \pi^{*}(\theta)<1\right\}\right)$, a contradiction. Thus $\phi^{*}(r) \geq \phi^{*}\left(r^{\prime}\right)$, and $\varphi^{*}=\phi^{*} \circ r^{*}$ is non-decreasing over $\left\{\theta \in[0,1] \mid \pi^{*}(\theta)<1\right\}$, as claimed.

\section{Appendix B}

Proof of Lemma 3. Suppose that $\pi^{*}(0) \neq \hat{\pi}(0,0)$ so that $z^{*}(0) \neq \hat{z}(0,0)$. We prove that, no matter the equilibrium belief function $\phi^{*}$, a seller of type 0 would be strictly better off 
choosing the symmetric information reserve price $(1-\lambda) \hat{z}(0,0)$ and getting expected utility $U\left(0, \phi^{*}((1-\lambda) \hat{z}(0,0)),(1-\lambda) \hat{z}(0,0)\right)$. Indeed, from (4), an easy computation shows that:

$$
U_{2}(0, \varphi, r)=\lambda\left\{[1-F(z(\varphi, r))]^{2}+\frac{2 r f(z(\varphi, r)) F(z(\varphi, r))}{1-\lambda}\right\}>0
$$

for any $(\varphi, r) \in[0,1]^{2}$. Hence $U(0, \varphi, r)$ is strictly increasing with respect to $\varphi$ for any reserve price $r$. Since $z^{*}(0) \neq \hat{z}(0,0)$, it follows that:

$$
\begin{aligned}
U\left(0, \phi^{*}((1-\lambda) \hat{z}(0,0)),(1-\lambda) \hat{z}(0,0)\right) \geq U(0,0,(1-\lambda) \hat{z}(0,0)) \\
=(1-\lambda) \int_{\hat{z}(0,0)}^{1}\left[\zeta-\frac{1-F(\zeta)}{f(\zeta)}\right] \mathrm{d} F(\zeta)^{2} \\
>(1-\lambda) \int_{z^{*}(0)}^{1}\left[\zeta-\frac{1-F(\zeta)}{f(\zeta)}\right] \mathrm{d} F(\zeta)^{2} \\
=\hat{U}\left(0,0, \pi^{*}(0)\right) \\
=U\left(0, \phi^{*}\left(r^{*}(0)\right), r^{*}(0)\right),
\end{aligned}
$$

which violates the sequential rationality condition, a contradiction.

Proof of Proposition 1. The mapping $\hat{U}$ satisfies assumptions (1)-(5) of Mailath (1987), with two exceptions. Specifically:

(1) $\hat{U} \in \mathcal{C}^{2}\left([0,1]^{2} \times \mathbb{R}\right)$;

(2) $\hat{U}_{2} \geq 0$;

(3) $\hat{U}_{13}>0$;

(4) For every $\theta \in[0,1]$, the equation $\hat{U}_{3}(\theta, \theta, \pi)=0$ has a unique solution $\hat{\pi}(\theta, \theta)$ in $\pi$, which maximizes $\hat{U}(\theta, \theta, \pi)$, and $\hat{U}_{33}(\theta, \theta, \hat{\pi}(\theta, \theta))<0$.

Condition (5) is emptily satisfied since $\hat{U}(\theta, \theta, \pi)$ is strictly concave with respect to $\pi$ for every $\theta \in[0,1]$. The only difference with Mailath's (1987) assumptions is that (2) may hold as an equality for $\pi=1$, while he assumes that $\hat{U}_{2}$ can never be equal to 0 .

The proof goes through a series of steps. Let $\pi^{*}$ be an incentive compatible separating allocation. From Mailath (1987, Appendix, Proposition 2), if $\pi^{*}$ is continuous at $\theta \in(0,1)$ and $\pi^{*}(\theta) \neq \hat{\pi}(\theta, \theta)$, then $\pi^{*}$ is differentiable at $\theta$ and:

$$
\frac{\mathrm{d} \pi^{*}}{\mathrm{~d} \theta}(\theta)=-\frac{\hat{U}_{2}\left(\theta, \theta, \pi^{*}(\theta)\right)}{\hat{U}_{3}\left(\theta, \theta, \pi^{*}(\theta)\right)}=\frac{\lambda\left[1-\pi^{*}(\theta)\right]}{(1-\lambda)\left[z^{*}(\theta)-\frac{1-F\left(z^{*}(\theta)\right)}{f\left(z^{*}(\theta)\right)}-\theta\right]}
$$

Note that Mailath's (1987) proof of this result does not require $\hat{U}_{2}$ to be strictly positive, and is thus valid in the present setting.

Next, if $\pi^{*}$ is continuous over some open interval $I \subset[0,1]$, then $\pi^{*}(\theta) \neq \hat{\pi}(\theta, \theta)$ for every $\theta \in I$. Indeed, suppose first that $\pi^{*}(\theta)=\hat{\pi}(\theta, \theta)$ for any $\theta$ in some open subinterval $E \subset I$. Then $\pi^{*}$ is differentiable over $E$ and, by the envelope condition (12), the derivative of $\hat{U}^{*}$ 
at any $\theta \in E$ is $\hat{\pi}(\theta, \theta)$. But since $\hat{U}^{*}(\theta)=\hat{U}(\theta, \theta, \hat{\pi}(\theta, \theta))$ for any $\theta \in E$, this derivative must also be equal to $\lambda+(1-\lambda) \hat{\pi}(\theta, \theta)$ by (14). For $\lambda>0$ and $\theta<1$, this is impossible, as $\hat{\pi}(\theta, \theta) \neq \lambda+(1-\lambda) \hat{\pi}(\theta, \theta)$. The rest of the proof then follows Mailath (1987, Appendix, Proposition 3$)$, using the fact that $\hat{U} \in \mathcal{C}^{2}\left([0,1]^{2} \times \mathbb{R}\right)$.

Proceeding as in Mailath (1987, Appendix, Proposition 4), one can then show that $\pi^{*}$ can be discontinuous at at most one point $\theta \in(0,1)$, and that if it were so, it would be strictly decreasing on one of $[0, \theta)$ or $(\theta, 1]$, and strictly increasing on the other. But this we know is impossible by Lemma 2 . Hence $\pi^{*}$ is continuous over $(0,1)$, so that $\pi^{*}(\theta) \neq \hat{\pi}(\theta, \theta)$ for every $\theta \in(0,1)$. From the first step of the proof, it follows that $\pi^{*}$ is differentiable and satisfies (35) over $(0,1)$. Furthermore, if $\pi^{*}$ satisfies the boundary condition (16), it is continuous over $[0,1]$, see Mailath (1987, Theorem 2).

We are thus led to study the initial boundary value problem (18). An argument parallel to Mailath (1987, Appendix, Proposition 5) implies that (18) has a unique non-decreasing solution $\pi^{S}$. What must be checked is that $\pi^{S}(\theta)<1$ for all $\theta<1$ and $\pi^{S}(1)=1$. (Note that this problem does not arise in Mailath (1987), since he assumes that $\hat{U}_{2}$ can never be equal to 0 .) To prove the first point, rewrite (35) as:

$$
\frac{\mathrm{d} \ln \left(1-F\left(z^{S}\right)^{2}\right)}{\mathrm{d} \theta}(\theta)=\frac{-\lambda}{(1-\lambda)\left[z^{S}(\theta)-\frac{1-F\left(z^{S}(\theta)\right)}{f\left(z^{S}(\theta)\right)}-\theta\right]} .
$$

Now if $\lim _{\theta \uparrow \bar{\theta}} z^{S}(\theta)=1$ for some $\bar{\theta} \in(0,1]$, then $\lim _{\theta \uparrow \bar{\theta}} \ln \left(1-F\left(z^{S}(\theta)\right)^{2}\right)=-\infty$, and by (36),

$$
\lim _{\theta \uparrow \bar{\theta}} z^{S}(\theta)-\frac{1-F\left(z^{S}(\theta)\right)}{f\left(z^{S}(\theta)\right)}-\theta=0
$$

or $\bar{\theta}=1$. Hence $\pi^{S}(\theta)<1$ for all $\theta<1$, as claimed. To prove the second point, note that since the right-derivative of $\pi^{S}$ at 0 is infinite as $z^{S}(0)=\hat{z}(0,0)$, while the mapping $\theta \mapsto \hat{\pi}(\theta, \theta)$ has a bounded derivative by assumption, one must have $\pi^{S}(\theta)>\hat{\pi}(\theta, \theta)$ for every $\theta>0$ close enough to 0 . This implies that there is no $\theta \in(0,1)$ such that $\pi^{S}(\theta)=\hat{\pi}(\theta, \theta)$. Indeed, suppose the contrary, and let $\bar{\theta}>0$ be the smallest of such points. Then $\pi^{S}(\theta)>\hat{\pi}(\theta, \theta)$ for any $\theta \in(0, \bar{\theta})$. However, the derivative of $\pi^{S}$ at $\bar{\theta}$ would be infinite by (35), while that of $\theta \mapsto \hat{\pi}(\theta, \theta)$ is bounded by assumption. Hence one would have $\pi^{S}(\theta)<\hat{\pi}(\theta, \theta)$ in a leftneighborhood of $\bar{\theta}$, a contradiction. It follows that (19) holds for every $\theta \in(0,1)$, and since $\hat{\pi}(\theta, \theta)$ converges to 1 as $\theta$ goes to 1 , one must have $\pi^{S}(1)=1$.

The last step of the proof consists to show that $\pi^{S}$ is incentive compatible. This can be done along the same lines as in Mailath (1987, Theorem 3), using the fact that $\hat{U}$ satisfies the single-crossing condition.

\section{Appendix C}

Proof of Proposition 2. Note first that there cannot be a bunching interval $(\bar{\theta}, 1]$ at the top of the seller's type distribution, in which the good is sold with strictly positive probability. Indeed, the expected selling price would be strictly less than 1 over $(\bar{\theta}, 1]$, and thus for $\varepsilon>0$ small enough, a seller with type $1-\varepsilon$ would be strictly better off not to sale by setting a reserve price of 1 . Note that this also implies that in any equilibrium, $\pi^{*}(\theta)$ goes to 1 as $\theta$ goes to 1 . Thus if a full bunching equilibrium exists, $\pi^{*}=1$ over $[0,1]$. But this is absurd since, no matter the buyers' beliefs, a seller of type $\theta=0$ would strictly gain by setting a reserve price $\hat{\pi}(0,0)$ and sell with positive probability. 
Proof of Proposition 3. We first establish the result over the interior of the set of separating types $S^{*}=\left\{\theta \in[0,1] \mid\left(r^{*}\right)^{-1}(r)=\{\theta\}\right.$ for some $\left.r \in[0,1]\right\}$. First, we prove that $\pi^{*}$ and $r^{*}$ are continuous over Int $S^{*}$. Clearly, $\varphi^{*}$ is continuous over $S^{*}$ as $\varphi^{*}(\theta)=\theta$ for each $\theta \in S^{*}$. Suppose that $r^{*}$ is discontinuous at $\theta \in \operatorname{Int} S^{*}$. Since $\hat{U}^{*}$ is continuous, a seller of type $\theta$ must be indifferent between $r^{*}\left(\theta^{-}\right)$and $r^{*}\left(\theta^{+}\right)$, and one must have $\hat{U}^{*}(\theta)=\hat{U}\left(\theta, \theta, \pi^{*}\left(\theta^{-}\right)\right)=$ $\hat{U}\left(\theta, \theta, \pi^{*}\left(\theta^{+}\right)\right)$. However, as $\phi^{*}$ is non-decreasing, each reserve price $r \in\left(r^{*}\left(\theta^{-}\right), r^{*}\left(\theta^{+}\right)\right)$is associated with the same out-of-equilibrium beliefs $\theta$. The strict concavity of $\hat{U}(\theta, \theta, \pi)$ with respect to $\pi \in\left(\pi^{*}\left(\theta^{-}\right), \pi^{*}\left(\theta^{+}\right)\right)$then implies that a seller of type $\theta$ could obtain a strictly greater payoff than $\hat{U}^{*}(\theta)$ by choosing a reserve price $r \in\left(r^{*}\left(\theta^{-}\right), r^{*}\left(\theta^{+}\right)\right)$, a contradiction. Hence, $r^{*}$ and thus $\pi^{*}$ are continuous at $\theta$, which implies the claim. Consider now $\theta \in \operatorname{Int} S^{*}$, and suppose that $\hat{\pi}(\theta, \theta)>\pi^{*}(\theta)$. Then, since $\hat{U}(\theta, \theta, \pi)$ is strictly concave with respect to $\pi$, it is also strictly increasing with respect to $\pi$ over $\left[\pi^{*}(\theta), \hat{\pi}(\theta, \theta)\right]$. For $\theta^{\prime}>\theta$ close enough to $\theta$, one has $\theta^{\prime} \in S^{*}$ and $\pi^{*}\left(\theta^{\prime}\right) \in\left[\pi^{*}(\theta), \hat{\pi}(\theta, \theta)\right]$ by continuity of $\pi^{*}$ at $\theta$. Therefore,

$$
\hat{U}\left(\theta, \varphi^{*}\left(\theta^{\prime}\right), \pi^{*}\left(\theta^{\prime}\right)\right)=\hat{U}\left(\theta, \theta^{\prime}, \pi^{*}\left(\theta^{\prime}\right)\right)>\hat{U}\left(\theta, \theta, \pi^{*}\left(\theta^{\prime}\right)\right) \geq \hat{U}\left(\theta, \theta, \pi^{*}(\theta)\right)=\hat{U}^{*}(\theta),
$$

where the strict inequality reflects the fact that $\hat{U}\left(\theta, \varphi, \pi^{*}\left(\theta^{\prime}\right)\right)$ is strictly increasing with respect to $\varphi$ since $\theta<1$ implies $\pi^{*}\left(\theta^{\prime}\right) \leq \hat{\pi}(\theta, \theta)<1$. As (37) violates (11), we obtain a contradiction. Therefore $\pi^{*}(\theta) \geq \hat{\pi}(\theta, \theta)$. That one cannot have $\pi^{*}(\theta)=\hat{\pi}(\theta, \theta)$ for some $\theta \in \operatorname{Int} S^{*}$ follows along the same lines as in the second step of the proof of Proposition 1 , using the fact that $\pi^{*}$ is continuous over Int $S^{*}$.

Next, let $\bar{\theta}$ be at the boundary of a bunching interval, with $r^{*}\left(\bar{\theta}^{-}\right)<r^{*}\left(\bar{\theta}^{+}\right)$. Then $z^{*}$ and $\varphi^{*}$ are also discontinuous at $\bar{\theta}$, with $\pi^{*}\left(\bar{\theta}^{-}\right)<\pi^{*}\left(\bar{\theta}^{+}\right)$and $\varphi^{*}\left(\bar{\theta}^{-}\right)<\varphi^{*}\left(\bar{\theta}^{+}\right)$. Moreover, $\hat{U}^{*}(\bar{\theta})=\hat{U}\left(\bar{\theta}, \varphi^{*}\left(\bar{\theta}^{-}\right), \pi^{*}\left(\bar{\theta}^{-}\right)\right)=\hat{U}\left(\bar{\theta}, \varphi^{*}\left(\bar{\theta}^{+}\right), \pi^{*}\left(\bar{\theta}^{+}\right)\right)$by continuity of $\hat{U}^{*}$. For any such $\bar{\theta}$,

$$
\pi^{*}\left(\bar{\theta}^{-}\right) \geq \hat{\pi}\left(\bar{\theta}, \varphi^{*}\left(\bar{\theta}^{-}\right)\right) .
$$

Indeed, suppose the contrary holds. Then, as $\hat{U}\left(\bar{\theta}, \varphi^{*}\left(\bar{\theta}^{-}\right), \pi^{*}\left(\bar{\theta}^{-}\right)\right)=\hat{U}\left(\bar{\theta}, \varphi^{*}\left(\bar{\theta}^{+}\right), \pi^{*}\left(\bar{\theta}^{+}\right)\right) \geq$ $\hat{U}\left(\bar{\theta}, \varphi^{*}\left(\bar{\theta}^{-}\right), \pi^{*}\left(\bar{\theta}^{+}\right)\right)$since $\hat{U}\left(\bar{\theta}, \varphi, \pi^{*}\left(\bar{\theta}^{+}\right)\right)$is non-decreasing with respect to $\varphi$, there exists some $\pi^{\prime} \in\left(\pi^{*}\left(\bar{\theta}^{-}\right), \pi^{*}\left(\bar{\theta}^{+}\right)\right]$such that:

$$
\hat{U}\left(\bar{\theta}, \varphi^{*}\left(\bar{\theta}^{-}\right), \pi^{\prime}\right)=\hat{U}\left(\bar{\theta}, \varphi^{*}\left(\bar{\theta}^{-}\right), \pi^{*}\left(\bar{\theta}^{-}\right)\right)=\hat{U}^{*}(\bar{\theta}) .
$$

It follows that for all $r \in\left(r^{*}\left(\bar{\theta}^{-}\right), r^{*}\left(\bar{\theta}^{+}\right)\right), \pi\left(\phi^{*}(r), r\right) \notin\left(\pi^{*}\left(\bar{\theta}^{-}\right), \pi^{\prime}\right)$. For otherwise, since $\phi^{*}(r) \geq \varphi^{*}\left(\bar{\theta}^{-}\right)$and $\hat{U}\left(\bar{\theta}, \varphi^{*}\left(\bar{\theta}^{-}\right), \pi\right)$ is strictly concave with respect to $\pi$, one would have:

$$
U\left(\bar{\theta}, \phi^{*}(r), r\right)=\hat{U}\left(\bar{\theta}, \phi^{*}(r), \pi\left(\phi^{*}(r), r\right)\right) \geq \hat{U}\left(\bar{\theta}, \varphi^{*}\left(\bar{\theta}^{-}\right), \pi\left(\phi^{*}(r), r\right)\right)>\hat{U}^{*}(\bar{\theta}),
$$

and the seller with type $\bar{\theta}$ would be strictly better off setting a reserve price $r$, a contradiction. Since $\pi\left(\phi^{*}\left(r^{*}\left(\bar{\theta}^{-}\right)\right), r^{*}\left(\bar{\theta}^{-}\right)\right)=\pi^{*}\left(\bar{\theta}^{-}\right)<\pi^{*}\left(\bar{\theta}^{+}\right)=\pi\left(\phi^{*}\left(r^{*}\left(\bar{\theta}^{+}\right)\right), r^{*}\left(\bar{\theta}^{+}\right)\right)$, it follows that there exists $r \in\left[r^{*}\left(\bar{\theta}^{-}\right), r^{*}\left(\bar{\theta}^{+}\right)\right]$such that $\pi\left(\phi^{*}\left(r^{-}\right), r\right)<\pi\left(\phi^{*}\left(r^{+}\right), r\right)$. But then $\phi^{*}\left(r^{-}\right)>\phi^{*}\left(r^{+}\right)$, which violates the monotonicity of $\phi^{*}$, a contradiction. Hence (38) holds, as claimed.

We can now establish the result over the interior of bunching intervals. Specifically, let $\theta$ belong to the interior of a bunching interval with right boundary $\bar{\theta}$, and assume without loss of generality that $\pi^{*}\left(\bar{\theta}^{-}\right)<1$. The mapping $(\pi, \vartheta) \mapsto \hat{U}\left(\vartheta, \varphi^{*}\left(\bar{\theta}^{-}\right), \pi\right)$ has increasing marginal returns as $\hat{U}_{13}=1$. Hence, by Edlin and Shannon (1998, Theorem 1$), \hat{\pi}\left(\vartheta, \varphi^{*}\left(\bar{\theta}^{-}\right)\right)$is strictly increasing with respect to $\vartheta$ and thus $\pi^{*}\left(\bar{\theta}^{-}\right) \geq \hat{\pi}\left(\bar{\theta}, \varphi^{*}\left(\bar{\theta}^{-}\right)\right)>\hat{\pi}\left(\theta, \varphi^{*}\left(\bar{\theta}^{-}\right)\right)$by (38). Finally, note that $\pi^{*}(\theta)=\pi^{*}\left(\bar{\theta}^{-}\right)$and $\varphi^{*}(\theta)=\varphi^{*}\left(\bar{\theta}^{-}\right)$. Therefore (21) holds, as claimed.

Proof of Corollary 1. For $\theta \in \operatorname{Int} S^{*}$, the result follows immediately from (21) as $\varphi^{*}(\theta)=\theta$. Now consider $\theta$ in the interior of a bunching interval, and let $\hat{\theta}$ in this interval be such that 
$\hat{\theta}=\varphi^{*}(\theta)$. The mapping $(\pi, \varphi) \mapsto \hat{U}(\theta, \varphi, \pi)$ has decreasing marginal returns as $\hat{U}_{23}=-\lambda$. Hence $\hat{\pi}(\theta, \varphi)$ is strictly decreasing with respect to $\varphi$. It follows that if $\theta \geq \hat{\theta}=\varphi^{*}(\theta)$, $\hat{\pi}\left(\theta, \varphi^{*}(\theta)\right) \geq \hat{\pi}(\theta, \theta)$, which implies $(22)$ as $\pi^{*}(\theta)>\hat{\pi}\left(\theta, \varphi^{*}(\theta)\right)$ by (21). Now suppose that $\theta<\hat{\theta}$. Since $(22)$ clearly holds at $\hat{\theta}$ by $(21)$ as $\varphi^{*}(\hat{\theta})=\hat{\theta}$, and $\pi^{*}(\hat{\theta})=\pi^{*}(\theta)$, we need only to prove that $\hat{\pi}(\theta, \theta) \leq \hat{\pi}(\hat{\theta}, \hat{\theta})$. To see this, note that the mapping $(\pi, \theta) \mapsto \hat{U}(\theta, \theta, \pi)$ has increasing marginal returns as $\hat{U}_{3}(\theta, \theta, \pi)$ is affine with respect to $\theta$, with a slope equal to $1-\lambda$. Hence $\hat{\pi}(\vartheta, \vartheta)$ is strictly increasing with respect to $\vartheta$, which implies the result.

Proof of Corollary 2. The proof goes through a series of steps. First, we set $\phi^{* *}=\phi^{*}$ over the range of $r^{*}$. Since $\left(\phi^{*}, r^{*}\right)$ is an equilibrium with monotone beliefs, $r^{*}$ is continuous on the interior of $S^{*}$, see the proof of Proposition 3. Hence, since $\phi^{*}\left(r^{*}(\theta)\right)=\theta$ for each $\theta \in S^{*}$, $\phi^{*}$ and thus $\phi^{* *}$ must be continuous on $r^{*}\left(\operatorname{Int} S^{*}\right)$.

Next, let $\bar{\theta}$ be at the boundary of a bunching interval, with $r^{*}\left(\bar{\theta}^{-}\right)<r^{*}\left(\bar{\theta}^{+}\right)$. Then $\pi^{*}\left(\bar{\theta}^{-}\right)<\pi^{*}\left(\bar{\theta}^{+}\right)$and $\varphi^{*}\left(\bar{\theta}^{-}\right)<\varphi^{*}\left(\bar{\theta}^{+}\right)$. Moreover, $\pi^{*}\left(\bar{\theta}^{-}\right) \geq \hat{\pi}\left(\bar{\theta}, \varphi^{*}\left(\bar{\theta}^{-}\right)\right)$by (38). We claim that $\pi^{*}\left(\bar{\theta}^{+}\right)<1$. For otherwise, $\hat{U}^{*}(\bar{\theta})=\bar{\theta}$, so that:

$$
\hat{U}\left(\bar{\theta}, \varphi^{*}\left(\bar{\theta}^{-}\right), \pi^{*}\left(\bar{\theta}^{-}\right)\right)=\bar{\theta}=\hat{U}\left(\bar{\theta}, \varphi^{*}\left(\bar{\theta}^{-}\right), 1\right),
$$

and it would follow from the strict concavity of $\hat{U}\left(\bar{\theta}, \varphi^{*}\left(\bar{\theta}^{-}\right), \pi\right)$ with respect to $\pi$ that $\pi^{*}\left(\bar{\theta}^{-}\right)<\hat{\pi}\left(\bar{\theta}, \varphi^{*}\left(\bar{\theta}^{-}\right)\right)$, in contradiction to (38). We now construct $\phi^{* *}$ over $\left(r^{*}\left(\bar{\theta}^{-}\right), r^{*}\left(\bar{\theta}^{+}\right)\right)$. For every $\varphi \in\left(\varphi^{*}\left(\bar{\theta}^{-}\right), \varphi^{*}\left(\bar{\theta}^{+}\right)\right)$, consider the following equation in $\pi$ :

$$
\hat{U}(\bar{\theta}, \varphi, \pi)=\hat{U}^{*}(\bar{\theta}) .
$$

Since $\pi^{*}\left(\bar{\theta}^{-}\right)<\pi^{*}\left(\bar{\theta}^{+}\right)<1, \hat{U}\left(\bar{\theta}, \varphi, \pi^{*}\left(\bar{\theta}^{-}\right)\right)$and $\hat{U}\left(\bar{\theta}, \varphi, \pi^{*}\left(\bar{\theta}^{+}\right)\right)$are strictly increasing with respect to $\varphi$. It follows that for any $\varphi \in\left(\varphi^{*}\left(\bar{\theta}^{-}\right), \varphi^{*}\left(\bar{\theta}^{+}\right)\right)$,

$$
\hat{U}\left(\bar{\theta}, \varphi, \pi^{*}\left(\bar{\theta}^{-}\right)\right)-\hat{U}^{*}(\bar{\theta})=\hat{U}\left(\bar{\theta}, \varphi, \pi^{*}\left(\bar{\theta}^{-}\right)\right)-\hat{U}\left(\bar{\theta}, \varphi^{*}\left(\bar{\theta}^{-}\right), \pi^{*}\left(\bar{\theta}^{-}\right)\right)>0
$$

and similarly:

$$
\hat{U}\left(\bar{\theta}, \varphi, \pi^{*}\left(\bar{\theta}^{+}\right)\right)-\hat{U}^{*}(\bar{\theta})=\hat{U}\left(\bar{\theta}, \varphi, \pi^{*}\left(\bar{\theta}^{+}\right)\right)-\hat{U}\left(\bar{\theta}, \varphi^{*}\left(\bar{\theta}^{+}\right), \pi^{*}\left(\bar{\theta}^{+}\right)\right)<0 .
$$

Thus, as $\hat{U}(\bar{\theta}, \varphi, \pi)$ is continuous with respect to $\pi,(39)$ has a solution in $\left(\pi^{*}\left(\bar{\theta}^{-}\right), \pi^{*}\left(\bar{\theta}^{+}\right)\right)$ for any $\varphi \in\left(\varphi^{*}\left(\bar{\theta}^{-}\right), \varphi^{*}\left(\bar{\theta}^{+}\right)\right)$. Note that for any such $\varphi, \hat{U}(\bar{\theta}, \varphi, \pi)>\hat{U}\left(\bar{\theta}, \varphi^{*}\left(\bar{\theta}^{-}\right), \pi\right)$ as long as $\pi<1$. Moreover, by $(38), \hat{U}\left(\bar{\theta}, \varphi^{*}\left(\bar{\theta}^{-}\right), \pi\right)$ reaches its maximum below $\pi^{*}\left(\bar{\theta}^{-}\right)$. Since $\hat{U}(\bar{\theta}, \varphi, \pi)$ is strictly concave with respect to $\pi$, it follows that (39) has a unique solution $\hat{\pi}^{*}(\varphi)$ that is continuous with respect to $\varphi$. One can then continuously extend $\hat{\pi}^{*}$ to $\left[\varphi^{*}\left(\bar{\theta}^{-}\right), \varphi^{*}\left(\bar{\theta}^{+}\right)\right]$ by setting $\hat{\pi}^{*}\left(\varphi^{*}\left(\bar{\theta}^{-}\right)\right)=\pi^{*}\left(\bar{\theta}^{-}\right)$and $\hat{\pi}^{*}\left(\varphi^{*}\left(\bar{\theta}^{+}\right)\right)=\pi^{*}\left(\bar{\theta}^{+}\right)$. Note that the mapping $\hat{\pi}^{*}$ is one-to-one over $\left(\varphi^{*}\left(\bar{\theta}^{-}\right), \varphi^{*}\left(\bar{\theta}^{+}\right)\right)$. Indeed, suppose that $\varphi^{*}\left(\bar{\theta}^{-}\right)<\varphi^{\prime}<\varphi^{\prime \prime}<\varphi^{*}\left(\bar{\theta}^{+}\right)$but $\hat{\pi}^{*}\left(\varphi^{\prime}\right)=\hat{\pi}^{*}\left(\varphi^{\prime \prime}\right)$. Then $\hat{U}\left(\bar{\theta}, \varphi^{\prime}, \hat{\pi}^{*}\left(\varphi^{\prime}\right)\right)=\hat{U}\left(\bar{\theta}, \varphi^{\prime \prime}, \hat{\pi}^{*}\left(\varphi^{\prime}\right)\right)$ by $(39)$, which contradicts the fact that $\hat{U}\left(\bar{\theta}, \varphi, \hat{\pi}^{*}\left(\varphi^{\prime}\right)\right)$ is strictly increasing with respect to $\varphi$ as $\hat{\pi}^{*}\left(\varphi^{\prime}\right)<\pi^{*}\left(\bar{\theta}^{+}\right)<1$. Hence, as $\hat{\pi}^{*}$ is continuous over $\left[\varphi^{*}\left(\bar{\theta}^{-}\right), \varphi^{*}\left(\bar{\theta}^{+}\right)\right]$and $\hat{\pi}^{*}\left(\varphi^{*}\left(\bar{\theta}^{-}\right)\right)=\pi^{*}\left(\bar{\theta}^{-}\right)<\pi^{*}\left(\bar{\theta}^{+}\right)=\hat{\pi}^{*}\left(\varphi^{*}\left(\bar{\theta}^{+}\right)\right)$, $\hat{\pi}^{*}$ is strictly increasing over $\left[\varphi^{*}\left(\bar{\theta}^{-}\right), \varphi^{*}\left(\bar{\theta}^{+}\right)\right]$. Thus $\hat{r}^{*}(\varphi)=\lambda \varphi+(1-\lambda)\left(F^{2}\right)^{-1}\left(\hat{\pi}^{*}(\varphi)\right)$ is continuous and strictly increasing with respect to $\varphi$ over this interval, with $\hat{r}^{*}\left(\varphi^{*}\left(\bar{\theta}^{-}\right)\right)=$ $r^{*}\left(\bar{\theta}^{-}\right)$and $\hat{r}^{*}\left(\varphi^{*}\left(\bar{\theta}^{+}\right)\right)=r^{*}\left(\bar{\theta}^{+}\right)$. Let $\phi^{* *}$ be the inverse of $\hat{r}^{*}$ over $\left.\left[r^{*}\left(\bar{\theta}^{-}\right), r^{*}\left(\bar{\theta}^{+}\right)\right)\right]$. Then $\phi^{* *}$ is strictly increasing and continuous over this interval, and so is $\hat{\pi}^{*} \circ \phi^{* *}$. It follows that the probability of no trade:

$$
\pi\left(\phi^{* *}(r), r\right)=F\left(\frac{r-\lambda \phi^{* *}(r)}{1-\lambda}\right)^{2}=F\left(\frac{\hat{r}^{*} \circ \phi^{* *}(r)-\lambda \phi^{* *}(r)}{1-\lambda}\right)^{2}=\hat{\pi}^{*} \circ \phi^{* *}(r)
$$


is also strictly increasing with respect to the reserve price $r$ over $\left[r^{*}\left(\bar{\theta}^{-}\right), r^{*}\left(\bar{\theta}^{+}\right)\right]$. The belief function $\phi^{* *}$ can be extended to the interval $\left[r^{*}(0), 1\right]$ by reiterating the above construction at any boundary of a bunching interval.

Now consider what happens at 0 . No matter the equilibrium belief function $\phi^{*}$, a seller with type 0 can always guarantee himself a payoff $\hat{U}(0,0, \hat{\pi}(0,0))$ by choosing the symmetric information reserve price $(1-\lambda) \hat{z}(0,0)$, see the proof of Lemma 3 . Hence one must have $\hat{U}^{*}(0) \geq \hat{U}(0,0, \hat{\pi}(0,0))$. Note that $\pi^{*}(0) \geq \hat{\pi}\left(0, \varphi^{*}(0)\right)$, with equality if the equilibrium is separating at 0 . In the latter case, one has $r^{*}(0)=(1-\lambda) \hat{z}(0,0)$ and one can let $\phi^{* *}$ be equal to 0 over $\left[0, r^{*}(0)\right]$ as in the separating equilibrium characterized in Section 3 . Suppose now that $\pi^{*}(0)>\hat{\pi}\left(0, \varphi^{*}(0)\right)$. Since $\hat{U}(0, \varphi, \pi)$ is strictly increasing with respect to $\varphi$ for $\pi<\pi^{*}(0)<1$ and strictly concave with respect to $\pi$, there exists a unique $\varphi_{0}<$ $\varphi^{*}(0)$ such that $\hat{U}^{*}(0)=\hat{U}\left(0, \varphi_{0}, \hat{\pi}\left(0, \varphi_{0}\right)\right)$. Proceeding as in the previous step of the proof, one can show that for any $\varphi \in\left[\varphi_{0}, \varphi^{*}(0)\right]$, the equation $\hat{U}(0, \varphi, \pi)=\hat{U}^{*}(0)$ has a unique solution $\hat{\pi}^{*}(\varphi)$, which is continuous and strictly increasing with respect to $\varphi$ over $\left[\varphi_{0}, \varphi^{*}(0)\right]$. Constructing $\hat{r}^{*}$ in the same way, we let $\phi^{* *}$ be the inverse of $\hat{r}^{*}$ over $\left[\hat{r}^{*}(0), r^{*}(0)\right]$, where $\hat{r}^{*}(0)=\lambda \varphi_{0}+(1-\lambda)\left(F^{2}\right)^{-1}\left(\hat{\pi}\left(0, \varphi_{0}\right)\right)$. Finally, we extend the belief function $\varphi^{* *}$ to the interval $\left[0, r^{*}(0)\right]$ by letting $\phi^{* *}$ be constant and equal to $\varphi_{0}$ over $\left[0, \hat{r}^{*}(0)\right]$.

The last step of the proof consist to show that $\left(\phi^{* *}, r^{*}\right)$ is an equilibrium. For any $\bar{\theta}$ at the boundary of a bunching interval, a seller of type $\bar{\theta}$ is indifferent between all reserve prices $r \in\left[r^{*}\left(\bar{\theta}^{-}\right), r^{*}\left(\bar{\theta}^{+}\right)\right]$given the belief function $\phi^{* *}$, since $\hat{U}\left(\bar{\theta}, \phi^{* *}(r), \pi\left(\phi^{* *}(r), r\right)\right)=\hat{U}^{*}(\bar{\theta})$ for all such $r$ by construction. We must therefore only check that a seller of type $\theta \neq \bar{\theta}$ does not strictly benefit from selecting a reserve price in $\left(r^{*}\left(\bar{\theta}^{-}\right), r^{*}\left(\bar{\theta}^{+}\right)\right)$. From $(11), \hat{U}^{*}(\theta) \geq$ $\hat{U}^{*}(\bar{\theta}+\varepsilon)+(\theta-\bar{\theta}-\varepsilon) \pi^{*}(\bar{\theta}+\varepsilon)$ for each $\varepsilon \in[-\bar{\theta}, 1-\bar{\theta}]$. As $\hat{U}^{*}$ is continuous, letting $\varepsilon$ go to 0 from below and from above yields:

$$
\hat{U}^{*}(\theta) \geq \hat{U}^{*}(\bar{\theta})+\max \left\{(\theta-\bar{\theta}) \pi^{*}\left(\bar{\theta}^{-}\right),(\theta-\bar{\theta}) \pi^{*}\left(\bar{\theta}^{+}\right)\right\} .
$$

For every $r \in\left(r^{*}\left(\bar{\theta}^{-}\right), r^{*}\left(\bar{\theta}^{+}\right)\right), \pi\left(\phi^{* *}(r), r\right) \in\left(\pi^{*}\left(\bar{\theta}^{-}\right), \pi^{*}\left(\bar{\theta}^{+}\right)\right)$by construction. Therefore,

$$
\begin{aligned}
\hat{U}^{*}(\theta) & \geq \hat{U}^{*}(\bar{\theta})+(\theta-\bar{\theta}) \pi\left(\phi^{* *}(r), r\right) \\
& =\hat{U}\left(\bar{\theta}, \phi^{* *}(r), \pi\left(\phi^{* *}(r), r\right)\right)+(\theta-\bar{\theta}) \pi\left(\phi^{* *}(r), r\right) \\
& =\hat{U}\left(\theta, \phi^{* *}(r), \pi\left(\phi^{* *}(r), r\right)\right) \\
& =U\left(\theta, \phi^{* *}(r), r\right),
\end{aligned}
$$

from which the result follows. Now consider what happens at 0 . A seller of type 0 is indifferent between all reserve prices $r \in\left[\hat{r}^{*}\left(0^{-}\right), r^{*}(0)\right]$ given the belief function $\phi^{* *}$, since $\hat{U}\left(0, \phi^{* *}(r), \pi\left(\phi^{* *}(r), r\right)\right)=\hat{U}^{*}(0)$ for all such $r$ by construction. Moreover, any $r<\hat{r}^{*}(0)$ leads to a probability of no trade strictly below $\hat{\pi}\left(0, \varphi_{0}\right)$. Given that $\phi^{* *}(r)=\varphi_{0}$ for any such $r$ and $\hat{U}^{*}(0)=\hat{U}\left(0, \varphi_{0}, \hat{\pi}\left(0, \varphi_{0}\right)\right)$ it follows that a seller of type 0 cannot improve upon $\hat{U}^{*}(0)$ by selecting a reserve price in $\left[0, \hat{r}^{*}(0)\right)$. We must therefore only check that a seller of type $\theta \neq 0$ does not strictly benefit from selecting a reserve price in $\left[0, r^{*}(0)\right)$. This can be done in a manner similar to above by noting that $\pi\left(\phi^{* *}(r), r\right) \in\left[0, \pi^{*}\left(0^{+}\right)\right)$and that $\hat{U}^{*}(0) \geq \hat{U}\left(0, \phi^{* *}(r), \pi\left(\phi^{* *}(r), r\right)\right)$ for any $r \in\left[0, r^{*}(0)\right)$. Hence the result.

Proof of Corollary 3. From the proof of Proposition 2, an equilibrium with a bunching interval $(\bar{\theta}, 1]$ at the top of the seller's type distribution must be such that $\pi^{*}(\theta)=1$ for every 
$\theta \in(\bar{\theta}, 1]$, and thus $\pi^{*}\left(\bar{\theta}^{+}\right)=1$. This implies that a seller with type $\bar{\theta}$ must be indifferent between selling with strictly positive probability and not selling at all. However, from the proof of Corollary 2, this is impossible under monotone beliefs, as this contradicts (38). Hence (23) holds, as claimed.

Now consider a separating equilibrium with monotone beliefs. From Lemma $3, \pi^{*}(0)=$ $\hat{\pi}(0,0)$. Moreover, from the proof of Proposition $3, \pi^{*}$ is continuous over $(0,1)$ and $\pi^{*}(\theta) \neq$ $\hat{\pi}(\theta, \theta)$ for any $\theta \in(0,1)$. From Mailath (1987, Appendix, Proposition 2), $\pi^{*}$ satisfies the differential equation (35) over $\left\{\theta \in[0,1] \mid \pi^{*}(\theta)<1\right\}$, and therefore coincides with $\pi^{S}$ on this interval as $\pi^{*}(0)=\pi^{S}(0)$. Since $\pi^{*}$ is continuous and $\pi^{S}(\theta)<1$ for all $\theta<1$, it follows that $\pi^{*}=\pi^{S}$, which implies the result.

Proof of Corollary 4. For any $\theta \in[0,1]$, let $z^{*}(\theta)=\left(F^{2}\right)^{-1}\left(\pi^{*}(\theta)\right)$ and $r^{*}(\theta)=\lambda \varphi^{*}(\theta)+$ $(1-\lambda) z^{*}(\theta)$. It follows from conditions (ii)-(iii) that $\pi^{*}$ is non-decreasing, and thus from condition (i) that $\varphi^{*}$ is non-decreasing. This implies that if $r^{*}(\theta)=r^{*}\left(\theta^{\prime}\right)$, then $\varphi^{*}(\theta)=\varphi\left(\theta^{\prime}\right)$ and $z^{*}(\theta)=z^{*}\left(\theta^{\prime}\right)$. One can therefore define a function $\phi^{*}$ over the range of $r^{*}$ by imposing that $\phi^{*}(r)=\varphi^{*}(\theta)$ whenever $r=r^{*}(\theta)$. The following sequential rationality condition holds for any $\left(\theta, \theta^{\prime}\right) \in[0,1]^{2}$ :

$$
U\left(\theta, \phi^{*}\left(r^{*}(\theta)\right), r^{*}(\theta)\right)=\hat{U}\left(\theta, \varphi^{*}(\theta), \pi^{*}(\theta)\right) \geq \hat{U}\left(\theta, \varphi^{*}\left(\theta^{\prime}\right), \pi^{*}\left(\theta^{\prime}\right)\right)=U\left(\theta, \phi^{*}\left(r^{*}\left(\theta^{\prime}\right)\right), r^{*}\left(\theta^{\prime}\right)\right),
$$

where the inequality is a standard consequence of conditions (ii)-(iii). To conclude, one needs only to extend the belief function $\phi^{*}$ to $[0,1]$, thereby allowing out-of-equilibrium reserve prices. Using conditions (iv)-(v), this can be done along the same lines as in the proof of Corollary 2.

Proof of Proposition 4. For any $\theta \in[0,1], \hat{U}_{3}(\theta, 0,1)=\theta-1+\lambda$. Since $\bar{\theta}>1-\lambda$ and $\hat{U}(\bar{\theta}, 0, \pi)$ is strictly concave with respect to $\pi$, it follows that it is also strictly increasing with respect to $\pi$, so that in particular $\hat{U}(\bar{\theta}, 0, \pi)<\hat{U}(\bar{\theta}, 0,1)=\bar{\theta}$ for any $\pi \in[0,1)$. As $\bar{\pi}<1$ and $\hat{U}(\bar{\theta}, \bar{\varphi}, \pi)$ is strictly concave with respect to $\pi$, condition (i) implies that $\bar{\pi}<\hat{\pi}(\bar{\theta}, \bar{\varphi})$. The only relevant aspect of the distribution $G$ will be that $\bar{\varphi}=\mathrm{E}[\tilde{\theta} \mid \tilde{\theta} \leq \bar{\theta}]$, which is consistent with the fact that $\bar{\varphi}<\bar{\theta}$. We denote $\overline{\bar{\varphi}}=\mathrm{E}[\tilde{\theta} \mid \tilde{\theta}>\bar{\theta}]$.

To construct the equilibrium, let $\bar{r}=\lambda \bar{\varphi}+(1-\lambda)\left(F^{2}\right)^{-1}(\bar{\pi})$, and set:

$$
r^{*}(\theta)=\left\{\begin{array}{l}
\bar{r} \quad \text { if } \quad \theta \leq \bar{\theta}, \\
1 \text { if } \theta>\bar{\theta} .
\end{array}\right.
$$

The associated $\pi^{*}$ is given by (24). Since a seller with type $\bar{\theta}$ is indifferent between not trading with probability $\bar{\pi}$ given beliefs $\bar{\varphi}$ and not trading at all by condition (i), the associated rent profile is continuous and given by:

$$
\hat{U}^{*}(\theta)=\left\{\begin{array}{cc}
\theta \bar{\pi}+\bar{\theta}(1-\bar{\pi}) & \text { if } \quad \theta \leq \bar{\theta}, \\
\theta & \text { if } \quad \theta>\bar{\theta} .
\end{array}\right.
$$

Let us define a belief function $\phi^{*}$ over $[\bar{r}, 1]$ as follows:

$$
\phi^{*}(r)=\left\{\begin{array}{ccc}
\bar{\varphi} & \text { if } & r=\bar{r}, \\
0 & \text { if } & \bar{r}<r<1, \\
\overline{\bar{\varphi}} & \text { if } & r=1 .
\end{array}\right.
$$


We now check that no seller's type has an incentive to deviate from the strategy (40) to a different reserve price $r \in[\bar{r}, 1]$ given the beliefs (42). Consider first a seller with type $\bar{\theta}$. By condition (i), he is indifferent between choosing $\bar{r}$ or 1 . If he deviates to some $r \in(\bar{r}, 1)$, then two cases may happen. Either $r>1-\lambda$ and the associated probability of no trade $\pi(0, r)$ is equal to 1 , in which case he gets the same utility than in the candidate equilibrium. Or $r<1-\lambda$ and the associated probability of no trade $\pi(0, r)$ is strictly smaller than 1 . In that case his utility is $\hat{U}(\bar{\theta}, 0, \pi)<\hat{U}(\bar{\theta}, 0, \pi)=\bar{\theta}=\hat{U}^{*}(\bar{\theta})$, and he strictly loses from deviating. Consider next a seller with type $\theta<\bar{\theta}$. Clearly, he has no incentive to deviate to a reserve price equal to 1 , as $\hat{U}^{*}(\theta)=\theta \bar{\pi}+\bar{\theta}(1-\bar{\pi})>\theta=\hat{U}(\theta, \overline{\bar{\varphi}}, 1)$ by (41). If he deviates to some $r \in(\bar{r}, 1)$, then two cases may happen. Either $r>1-\lambda$ and the associated probability of no trade $\pi(0, r)$ is equal to 1 , in which case he gets again a lower utility than in the candidate equilibrium. Or $r<1-\lambda$ and the associated probability of no trade $\pi(0, r)$ is strictly larger than $\bar{\pi}$ as $r>\bar{r}$ and $\bar{\varphi}>0$. His utility from this deviation is then:

$$
\begin{aligned}
\hat{U}(\theta, 0, \pi(0, r)) & =\hat{U}(\bar{\theta}, 0, \pi(0, r))+(\theta-\bar{\theta}) \pi(0, r) \\
& <\hat{U}(\bar{\theta}, 0, \pi(0, r))+(\theta-\bar{\theta}) \bar{\pi} \\
& <\bar{\theta}+(\theta-\bar{\theta}) \bar{\pi} \\
& =\hat{U}(\bar{\theta}, \bar{\varphi}, \bar{\pi})+(\theta-\bar{\theta}) \bar{\pi} \\
& =\hat{U}(\theta, \bar{\varphi}, \bar{\pi}) \\
& =\hat{U}^{*}(\theta)
\end{aligned}
$$

where the first inequality follows from $\theta<\bar{\theta}$ and $\pi(0, r)>\bar{\pi}$, and the second from the fact that $\hat{U}(\bar{\theta}, 0, \pi)$ is strictly increasing with respect to $\pi$. This implies that a seller with type $\theta<\bar{\theta}$ has no incentive to deviate to some $r \in(\bar{r}, 1)$. Consider last a seller with type $\theta>\bar{\theta}$. He has no incentive to deviate to a reserve price $\bar{r}$, as he would thereby get $\hat{U}(\theta, \bar{\varphi}, \bar{\pi})=\theta \bar{\pi}+\bar{\theta}(1-\bar{\pi})<\theta=\hat{U}^{*}(\theta)$. Moreover, since $\theta>\bar{\theta}>1-\lambda, U(\theta, 0, \pi)$ is strictly increasing with respect to $\pi$. It follows that, as for a seller with type $\bar{\theta}$, a seller with type $\theta>\bar{\theta}$ has no incentive to deviate to a reserve price $r \in(\bar{r}, 1)$.

It remains to construct the belief function $\phi^{*}$ over $[0, \bar{r})$. Two cases must be distinguished. If $\bar{\pi} \leq \hat{\pi}(0, \bar{\varphi})$, then we let $\phi^{*}$ be equal to $\bar{\varphi}$ over $[0, \bar{r})$. Since $\hat{\pi}(\theta, \bar{\varphi})$ is strictly increasing with respect to $\theta$, it follows that for each $\theta \in[0, \bar{\theta}]$, the mapping $\hat{U}(\theta, \bar{\varphi}, \pi)$ is strictly increasing with respect to $\pi$ over $[0, \bar{\pi}]$. Hence no seller's type $\theta \in[0, \bar{\theta}]$ has an incentive to choose a reserve price $r<\bar{r}$, as this would only decrease the probability of no trade $\pi(\bar{\varphi}, r)$ relative to $\bar{\pi}=\pi(\bar{\varphi}, \bar{r})$. Finally, a seller with type $\theta>\bar{\theta}$ has no incentive to deviate to some $r<\bar{r}$, as he would thereby get:

$$
\hat{U}(\theta, \bar{\varphi}, \pi(\bar{\varphi}, r))=\hat{U}(\bar{\theta}, \bar{\varphi}, \pi(\bar{\varphi}, r))+(\theta-\bar{\theta}) \bar{\pi}(\bar{\varphi}, r)<\bar{\theta}+(\theta-\bar{\theta}) \pi(\bar{\varphi}, r)<\theta=\hat{U}^{*}(\theta) .
$$

Thus $\left(\phi^{*}, r^{*}\right)$ is an equilibrium of the informed seller game. Next, if $\bar{\pi}>\pi(0, \bar{\varphi})$, one can construct $\phi^{*}$ over $[0, \bar{r})$ as in the proof of Corollary 2, using condition (ii). Specifically, there exists some $\hat{r}^{*}(0)<\bar{r}$ such that one can construct $\phi^{*}$ over $\left[\hat{r}^{*}(0), \bar{r}\right]$ so as to make a seller with type 0 indifferent between all reserve prices $r \in\left[\hat{r}^{*}(0), \bar{r}\right]$. One then extend $\phi^{*}$ to the interval 
$[0, \bar{r}]$ by letting $\phi^{*}$ be constant and equal to $\phi^{*}\left(\hat{r}^{*}(0)\right)$ over $\left[0, \hat{r}^{*}(0)\right)$. The construction ensures that $\hat{U}^{*}(0)=\hat{U}\left(0, \phi^{*}\left(\hat{r}^{*}(0)\right), \hat{\pi}\left(0, \phi^{*}\left(\hat{r}^{*}(0)\right)\right)\right)$, so that a seller of type 0 cannot improve upon $\hat{U}^{*}(0)$ by selecting a reserve price in $\left[0, \hat{r}^{*}(0)\right)$, and that the probability of no trade $\pi\left(\phi^{*}(r), r\right)$ is a non-decreasing function of $r$ over $[0, \bar{r}]$. To conclude, we must therefore only check that a seller of type $\theta \neq 0$ does not strictly benefit from selecting a reserve price in $[0, \bar{r})$. This can be done along the lines of the proof of Corollary 2 by noting that $\hat{U}^{*}(\theta) \geq \hat{U}^{*}(0)+\theta \bar{\pi}$ from (41), along with $\pi\left(\phi^{* *}(r), r\right) \in[0, \bar{\pi})$ and $\hat{U}^{*}(0) \geq \hat{U}\left(0, \phi^{* *}(r), \pi\left(\phi^{* *}(r), r\right)\right)$ for any $r \in[0, \hat{r})$. Thus $\left(\phi^{*}, r^{*}\right)$ is an equilibrium of the informed seller game, and the result follows.

Proof of Lemma 4. Since $\pi^{*}\left(\bar{\theta}^{+}\right)<1$ in an equilibrium with bunching below $\bar{\theta}$ at $\bar{\pi}$, and $\hat{U}(\bar{\theta}, \bar{\theta}, 1)=\bar{\theta}$, condition (25) can be rewritten as:

$$
\hat{U}(\bar{\theta}, \bar{\varphi}(\bar{\theta}), \bar{\pi})>\bar{\theta} .
$$

Conditions (26)-(27) and (43) are necessary and sufficient for an equilibrium with bunching below $\bar{\theta}$ at $\bar{\pi}$ to exist. Since $\hat{\pi}(0, \bar{\varphi}(\bar{\theta}))<\hat{\pi}(\bar{\theta}, \bar{\varphi}(\bar{\theta})) \leq \bar{\pi}$, the left-hand sides of (26) and (43) are decreasing with respect to $\bar{\pi}$. Therefore, if these conditions are met at $\bar{\pi}>\hat{\pi}(\bar{\theta}, \bar{\varphi}(\bar{\theta}))$, they are also met at $\hat{\pi}(\bar{\theta}, \bar{\varphi}(\bar{\theta}))$. As a result, a $[0, \bar{\theta}]$-equilibrium exists. Denote by $\pi^{*}$ and $\pi_{\bar{\theta}}$ the probabilities of no trade in the initial equilibrium and in the $[0, \bar{\theta}]$-equilibrium, respectively. By construction, $\pi_{\bar{\theta}}<\pi^{*}$ over $[0, \bar{\theta}]$. Moreover, since $\hat{U}(\bar{\theta}, \bar{\varphi}(\bar{\theta}), \bar{\pi})<\hat{U}(\bar{\theta}, \bar{\varphi}(\bar{\theta}), \hat{\pi}(\bar{\theta}, \bar{\varphi}(\bar{\theta})))$, (26) implies that $\hat{U}\left(\bar{\theta}, \bar{\theta}, \pi^{*}\left(\bar{\theta}^{+}\right)\right)<\hat{U}\left(\bar{\theta}, \bar{\theta}, \pi_{\bar{\theta}}\left(\bar{\theta}^{+}\right)\right)$, so that $\pi_{\bar{\theta}}\left(\bar{\theta}^{+}\right)<\pi^{*}\left(\bar{\theta}^{+}\right)$as both $\pi_{\bar{\theta}}\left(\bar{\theta}^{+}\right)$ and $\pi^{*}\left(\bar{\theta}^{+}\right)$are larger than $\hat{\pi}(\bar{\theta}, \bar{\theta}) \leq \hat{\pi}(\bar{\theta}, \bar{\varphi}(\theta))$. Since the solutions to the differential equation (17) are ranked by their initial conditions, $\pi_{\bar{\theta}}<\pi^{*}$ over $(\bar{\theta}, 1)$. Finally, denote by $\hat{U}^{*}$ and $\hat{U}_{\bar{\theta}}$ the surplus of the seller in the initial equilibrium and in the $[0, \bar{\theta}]$-equilibrium, respectively. From the envelope condition (12) and the fact that $\hat{U}^{*}(1)=\hat{U}_{\bar{\theta}}(1)=1$, one has:

$$
\hat{U}^{*}(\theta)=1-\int_{\theta}^{1} \pi^{*}(\vartheta) \mathrm{d} \vartheta<1-\int_{\theta}^{1} \pi_{\bar{\theta}}(\vartheta) \mathrm{d} \vartheta=\hat{U}_{\bar{\theta}}(\theta)
$$

for every $\theta \in[0,1)$, and the result follows.

Proof of Proposition 5. For large enough $\bar{\theta}$, the result follows from the remark before the statement of the result. Next, $\hat{U}(0, \bar{\varphi}(0), \hat{\pi}(0, \bar{\varphi}(0)))=\hat{U}(0,0, \hat{\pi}(0,0))>0$, and:

$$
\frac{\mathrm{d} \hat{U}}{\mathrm{~d} \bar{\theta}}(0, \bar{\varphi}(\bar{\theta}), \hat{\pi}(\bar{\theta}, \bar{\varphi}(\bar{\theta})))=\lambda[1-\hat{\pi}(\bar{\theta}, \bar{\varphi}(\bar{\theta}))] \frac{\mathrm{d} \bar{\varphi}}{\mathrm{d} \bar{\theta}}(\bar{\theta})-\bar{\theta} \frac{\mathrm{d} \hat{\pi}}{\mathrm{d} \bar{\theta}}(\bar{\theta}, \bar{\varphi}(\bar{\theta})),
$$

which is strictly positive at $\bar{\theta}=0$. Thus conditions (28)-(29) are satisfied for $\bar{\theta}$ small enough, which implies the result.

\section{Appendix D}

Proof of Lemma 5. Standard computations imply that, for each $i=1,2$,

$$
\begin{aligned}
\mathrm{E}\left[U^{B}(\tilde{\theta})\right] & =1-\mathrm{E}\left[\frac{G(\tilde{\theta})}{g(\tilde{\theta})} p(\tilde{\mathcal{T}})\right], \\
\mathrm{E}\left[U^{B, i}\left(\tilde{\zeta}^{i}\right)\right] & =(1-\lambda) \mathrm{E}\left[\frac{1-F\left(\tilde{\zeta}^{i}\right)}{f\left(\tilde{\zeta}^{i}\right)} p^{i}(\tilde{\mathcal{T}})\right] .
\end{aligned}
$$


The broker's problem becomes thus to maximize:

$$
\mathrm{E}\left[p(\tilde{\mathcal{T}})\left[\tilde{\theta}+\frac{G(\tilde{\theta})}{g(\tilde{\theta})}\right]+\sum_{i=1}^{2} p^{i}(\tilde{\mathcal{T}})\left\{\lambda \tilde{\theta}+(1-\lambda)\left[\tilde{\zeta}^{i}-\frac{1-F\left(\tilde{\zeta}^{i}\right)}{f\left(\tilde{\zeta}^{i}\right)}\right]\right\}\right]
$$

under the feasibility constraint. The result follows immediately.

Proof of Proposition 6. We first prove that for any $\varepsilon \in(0,1)$,

$$
\Delta^{\mathcal{P}} \equiv 1-\int_{0}^{1} F(\hat{z}(\theta))^{2} g(\theta) \mathrm{d} \theta-\mathcal{P}^{B} \leq \mathcal{O}(\varepsilon) .
$$

when $G$ is $\varepsilon$-concentrated at $\theta_{0} \in(\varepsilon, 1-\varepsilon)$, where $\mathcal{O}(\varepsilon) / \varepsilon$ is bounded in a neighborhood of 0 . Indeed, using the expression for $\mathcal{P}^{B}$, and letting $\bar{f}=\max _{\theta \in[0,1]} f(\theta)$ and $\bar{z}=\sup _{\theta \in[0,1)} \hat{z}^{\prime}(\theta)$, one obtains:

$$
\begin{aligned}
\Delta^{\mathcal{P}} & =\int_{0}^{1}\left[F\left(\hat{z}\left(\theta+\frac{G(\theta)}{(1-\lambda) g(\theta)}\right)\right)^{2}-F(\hat{z}(\theta))^{2}\right] g(\theta) \mathrm{d} \theta \\
& \leq 2 \bar{f} \int_{0}^{1}\left[\hat{z}\left(\theta+\frac{G(\theta)}{(1-\lambda) g(\theta)}\right)-\hat{z}(\theta)\right] g(\theta) \mathrm{d} \theta \\
& \leq 2 \bar{f}\left[\int_{0}^{\theta_{0}-\varepsilon} g(\theta) \mathrm{d} \theta+\bar{z} \int_{\theta_{0}-\varepsilon}^{\theta_{0}+\varepsilon} \frac{G(\theta)}{1-\lambda} \mathrm{d} \theta+\int_{\theta_{0}+\varepsilon}^{1} g(\theta) \mathrm{d} \theta\right] \\
& \leq 2 \bar{f} \varepsilon\left(1+\frac{2 \bar{z}}{1-\lambda}\right),
\end{aligned}
$$

which implies (44). It follows that:

$$
\mathcal{P}^{S}-\mathcal{P}^{B} \leq \int_{0}^{1}\left[F(\hat{z}(\theta))^{2}-F\left(z^{S}(\theta)\right)^{2}\right] g(\theta) \mathrm{d} \theta+\mathcal{O}(\varepsilon) .
$$

By Proposition $1, z^{S}(\theta)>\hat{z}(\theta)$ for all $\theta \in(0,1)$. Hence,

$$
\begin{aligned}
\mathcal{P}^{S}-\mathcal{P}^{B} & \leq \int_{\theta_{0}-\varepsilon}^{\theta_{0}+\varepsilon}\left[F(\hat{z}(\theta))^{2}-F\left(z^{S}(\theta)\right)^{2}\right] \mathrm{d} \theta+\mathcal{O}(\varepsilon) \\
& \leq\left[F\left(\hat{z}\left(\theta_{0}+\varepsilon\right)\right)^{2}-F\left(z^{S}\left(\theta_{0}-\varepsilon\right)\right)^{2}\right]\left[G\left(\theta_{0}+\varepsilon\right)-G\left(\theta_{0}-\varepsilon\right)\right]+\mathcal{O}(\varepsilon) \\
& \leq\left[F\left(\hat{z}\left(\theta_{0}+\varepsilon\right)\right)^{2}-F\left(z^{S}\left(\theta_{0}-\varepsilon\right)\right)^{2}\right](1-\varepsilon)+\mathcal{O}(\varepsilon) \\
& <0
\end{aligned}
$$

whenever $\varepsilon>0$ is small enough. The result follows.

Proof of Proposition 7. We first prove that for any $\varepsilon \in(0,1)$,

$$
\Delta^{\mathcal{S}} \equiv \mathrm{E}[\tilde{\theta}]+2(1-\lambda) \int_{0}^{1} \int_{\hat{z}(\theta)}^{1} F(\zeta)(\zeta-\theta) f(\zeta) \mathrm{d} \zeta g(\theta) \mathrm{d} \theta-\mathcal{S}^{B} \leq \mathcal{O}(\varepsilon)
$$

whenever $G$ is $\varepsilon$-concentrated at $\theta_{0} \in(\varepsilon, 1-\varepsilon)$, where $\mathcal{O}(\varepsilon) / \varepsilon$ is bounded in a neighborhood of 0 . Indeed, using the expression for $\mathcal{S}^{B}$, and defining $\bar{f}$ and $\bar{z}$ as in the proof of Proposition 6 , one obtains: 


$$
\begin{aligned}
\Delta^{\mathcal{S}} & =2(1-\lambda) \int_{0}^{1} \int_{\hat{z}(\theta)}^{\hat{z}\left(\theta+\frac{G(\theta)}{(1-\lambda) g(\theta)}\right)} F(\zeta)(\zeta-\theta) f(\zeta) \mathrm{d} \zeta g(\theta) \mathrm{d} \theta \\
& \leq 2(1-\lambda) \bar{f}\left\{\int_{0}^{\theta_{0}-\varepsilon} g(\theta) \mathrm{d} \theta+\int_{\theta_{0}-\varepsilon}^{\theta_{0}+\varepsilon}\left[\hat{z}\left(\theta+\frac{G(\theta)}{(1-\lambda) g(\theta)}\right)-\hat{z}(\theta)\right] g(\theta) \mathrm{d} \theta+\int_{\theta_{0}+\varepsilon}^{1} g(\theta) \mathrm{d} \theta\right\} \\
& \leq 2(1-\lambda) \bar{f}\left[\int_{0}^{\theta_{0}-\varepsilon} g(\theta) \mathrm{d} \theta+\bar{z} \int_{\theta_{0}-\varepsilon}^{\theta_{0}+\varepsilon} \frac{G(\theta)}{1-\lambda} \mathrm{d} \theta+\int_{\theta_{0}+\varepsilon}^{1} g(\theta) \mathrm{d} \theta\right] \\
& \leq 2(1-\lambda) \bar{f} \varepsilon\left(1+\frac{2 \bar{z}}{1-\lambda}\right)
\end{aligned}
$$

which implies (45). It follows that:

$$
\mathcal{S}^{S}-\mathcal{S}^{B} \leq-2(1-\lambda) \int_{0}^{1} \int_{\hat{z}(\theta)}^{z^{S}(\theta)} F(\zeta)(\zeta-\theta) f(\zeta) \mathrm{d} \zeta g(\theta) \mathrm{d} \theta+\mathcal{O}(\varepsilon) .
$$

By Proposition $1, z^{S}(\theta)>\hat{z}(\theta)$ for all $\theta \in(0,1)$. Hence, letting $m(\theta)$ be the minimum of $F(\zeta)(\zeta-\theta) f(\zeta)$ with respect to $\zeta \in\left[\hat{z}(\theta), z^{S}(\theta)\right]$, which is bounded away from 0 on $\left[\theta_{0}-\varepsilon, \theta_{0}+\varepsilon\right]$,

$$
\begin{aligned}
\mathcal{S}^{S}-\mathcal{S}^{B} & \leq-2(1-\lambda) \int_{\theta_{0}-\varepsilon}^{\theta_{0}+\varepsilon} \int_{\hat{z}(\theta)}^{z^{S}(\theta)} F(\zeta)(\zeta-\theta) f(\zeta) \mathrm{d} \zeta g(\theta) \mathrm{d} \theta+\mathcal{O}(\varepsilon) \\
& \leq 2(1-\lambda) \int_{\theta_{0}-\varepsilon}^{\theta_{0}+\varepsilon} m(\theta)\left[\hat{z}(\theta)-z^{S}(\theta)\right] g(\theta) \mathrm{d} \theta+\mathcal{O}(\varepsilon) \\
& \leq 2(1-\lambda) \min _{\theta \in\left[\theta_{0}-\varepsilon, \theta_{0}+\varepsilon\right]}\{m(\theta)\}\left[\hat{z}\left(\theta_{0}+\varepsilon\right)-z^{S}\left(\theta_{0}-\varepsilon\right)\right]\left[G\left(\theta_{0}+\varepsilon\right)-G\left(\theta_{0}-\varepsilon\right)\right]+\mathcal{O}(\varepsilon) \\
& \leq 2(1-\lambda) \min _{\theta \in\left[\theta_{0}-\varepsilon, \theta_{0}+\varepsilon\right]}\{m(\theta)\}\left[\hat{z}\left(\theta_{0}+\varepsilon\right)-z^{S}\left(\theta_{0}-\varepsilon\right)\right](1-\varepsilon)+\mathcal{O}(\varepsilon) \\
& <0
\end{aligned}
$$

whenever $\varepsilon>0$ is small enough. The result follows. 


\section{References}

Akerlof, G., 1970. The market for 'lemons': qualitative uncertainty and the market mechanism. Quart. J. Econ. 89, 488-500.

Biglaiser, G., 1993. Middlemen as experts. RAND J. Econ. 24, 212-223.

Cai, H., Riley, J., Ye, L., 2002. Reserve price signaling. Mimeo, UCLA.

Caillaud, B., Jullien, B., 2003. Chicken and egg: competition among intermediation service providers. RAND J. Econ. 34, 521-552.

Dellarocas, C., 2004. Sanctioning reputation mechanisms in online trading environments with moral hazard. MIT Sloan Working Paper 4297-03.

DeMarzo, P., Duffie, D., 1999. A liquidity-based model of security design. Econometrica 67, 65-99.

Diamond, D., 1984. Financial intermediation and delegated monitoring. Rev. Econ. Stud. $51,393-414$.

Edlin, A.S., Shannon, C., 1998. Strict monotonicity in comparative statics. J. Econ. Theory $81,201-219$.

Glosten, L.R., 1994. Is the electronic open limit order book inevitable? J. Finance 49, $1127-1161$.

Jullien, B., Mariotti, T., 2003. Auction and the informed seller problem. IDEI Working Paper.

Laffont, J.-J., Maskin, E., 1990. The efficient market hypothesis and insider trading on the stock market. J. Polit. Economy 98, 70-93.

Leland, H., Pyle, D., 1977. Information asymmetries, financial structure and financial intermediaries. J. Finance 32, 371-387.

Lizzeri, A., 1999. Information revelation and certification information. RAND J. Econ. 30, 214-231.

Mailath, G.J., 1987. Incentive compatibility in signaling games with a continuum of types. Econometrica 55, 1349-1365.

Mailath, G.J., Okuno-Fujiwara, M., Postlewaite, A., 1993. Belief-based refinements in signalling games. J. Econ. Theory 60, 241-276.

Maskin, E., Tirole, J., 1992. The principal-agent relationship with an informed principal, II: common values. Econometrica 60, 1-42.

Milgrom, P., Weber, R., 1982. A theory of auctions and competitive bidding. Econometrica $50,1089-1122$.

Myerson, R.B., Satterthwaite, M.A., 1983. Efficient mechanisms for bilateral trading. J. Econ. Theory 29, 265-281. 
Peyrache, E., Quesada, L., 2003. Strategic certification. Mimeo, HEC and University of Wisconsin-Madison.

Riley, J., Samuelson, W., 1981. Optimal auctions. Amer. Econ. Rev. 71, 381-392.

Rochet, J.-C., Tirole, J., 2003. Platform competition in two-sided markets. J. Europ. Econ. Assoc. 1, 990-1029.

Rubinstein, A., Wolinsky, A., 1987. Middlemen. Quart. J. Econ. 102, 581-593.

Yavas, A., 1994. Middlemen in bilateral search markets. J. Lab. Econ. 12, 406-429. 


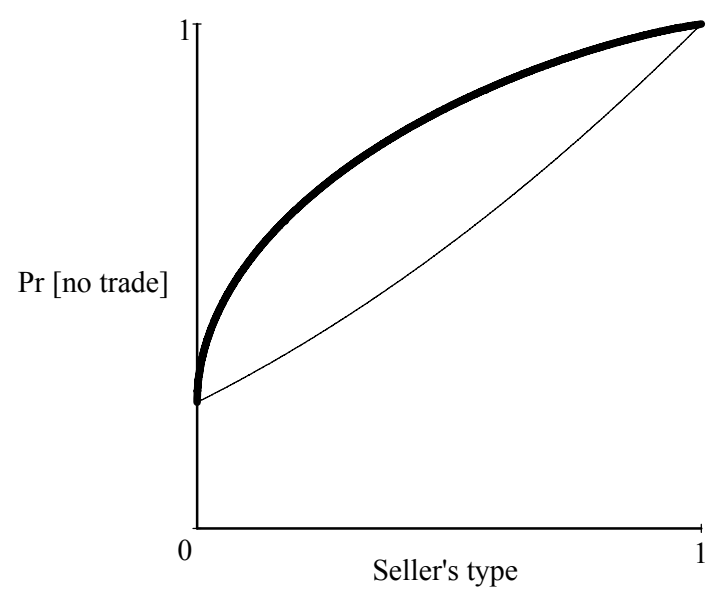

Fig. 1. Probabilities of no trade in the separating equilibrium and under symmetric information.

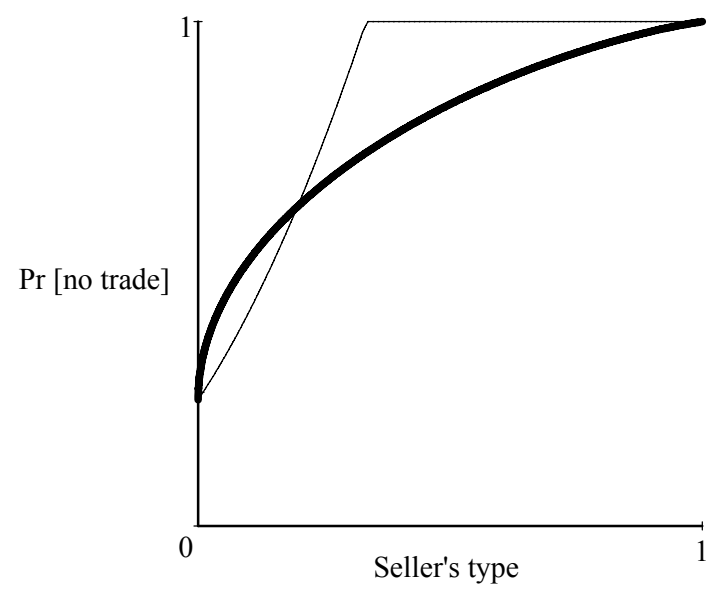

Fig. 2. Probabilities of no trade in the separating equilibrium and in the monopoly broker allocation.

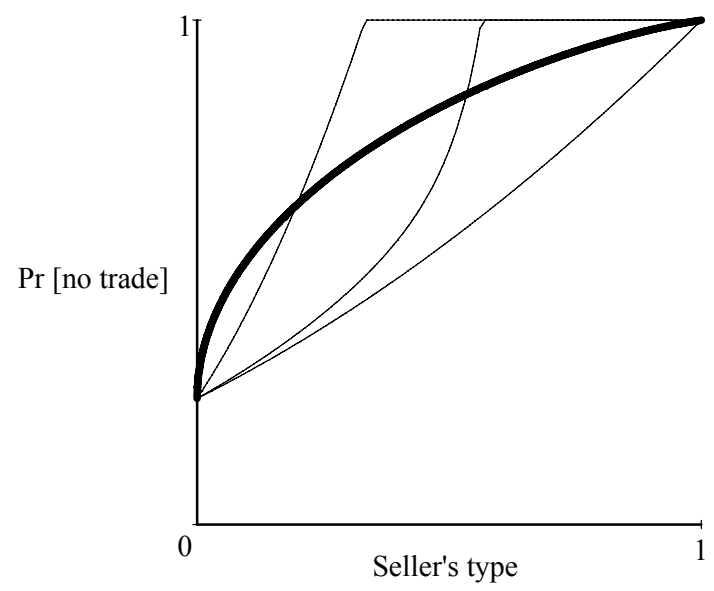

Fig. 3. Impact of increased concentration of the seller's type distribution. 